\title{
Vertical structure of mean cross-shore currents across a barred surf zone
}

\author{
John W. Haines and Asbury H. Sallenger, Jr. \\ U.S. Geological Survey, Center for Coastal Geology, St. Petersburg, Florida
}

\begin{abstract}
Mean cross-shore currents observed across a barred surf zone are compared to model predictions. The model is based on a simplified momentum balance with a turbulent boundary layer at the bed. Turbulent exchange is parameterized by an eddy viscosity formulation, with the eddy viscosity $A_{v}$ independent of time and the vertical coordinate. Mean currents result from gradients due to wave breaking and shoaling, and the presence of a mean setup of the free surface. Descriptions of the wave field are provided by the wave transformation model of Thornton and Guza [1983]. The wave transformation model adequately reproduces the observed wave heights across the surf zone. The mean current model successfully reproduces the observed cross-shore flows. Both observations and predictions show predominantly offshore flow with onshore flow restricted to a relatively thin surface layer. Successful application of the mean flow model requires an eddy viscosity which varies horizontally across the surf zone. Attempts are made to parameterize this variation with some success. The data does not discriminate between alternative parameterizations proposed. The overall variability in eddy viscosity suggested by the model fitting should be resolvable by field measurements of the turbulent stresses. Consistent shortcomings of the parameterizations, and the overall modeling effort, suggest avenues for further development and data collection.
\end{abstract}

\section{Introduction}

Mean cross-shore flows have been identified as a critical factor in the evolution and maintenance of the nearshore profile [Bowen, 1980; Dally and Dean, 1984]. Complete models of these flows, suitable for sediment transport modeling, would include detailed specification of the bottom boundary layer, including bed shear stresses. Available field data in the surf zone are linited to observations of the interior flow, relatively far from the bed. It is this interior flow, often characterized as underton, which we address here. This restricted outlook still provides information relevant to suspended sediment transport and profile evolution within the surf zone.

Theoretical models of cross-shore currents generated by normally incident waves on straight coasts have significantly improved our understanding of undertow and cross-shore circulation within the surf zone [Svendsen and Lorenz, 1989]. Numerous studies [Borecki, 1982; DeVriend and Stive, 1987; Dally, 1980; Diegarrd et al., 1991; Hansen and Svendsen, 1984; Stive and Wind, 1986; Svendsen et al., 1987; Svendsen and Lorenz, 1987] have addressed the dynamics of such systems in some

This paper is not subject to U.S. copyright. Published in 1994 by the American Geophysical Union.

Paper number 94JC00427. detail. These modeling efforts, with some laboratory verification, have far outstripped efforts to provide supportive field data. The models, as posed, require specification of the dynamical forcing terms with an accuracy not provided by existing field data sets. Application of such models to field conditions awaits improved data and field-verified determination of the parameters of the models. Svendsen et al. [1987, p. 11,856] indicated as much when they stated:

There is a need for further experimental investigation which can illustrate the variations of [the eddy viscosity] with wave conditions and bottom topography. Also, field measurements are much needed provided they include sufficient information also about wave heights and their attenuation, the setup gradient, etc., in addition to the actual turbulence measurements.

This ideal data set does not yet exist. In particular, setup has not been measured simultaneously with detailed wave observations. Turbulence measurements in the surf zone are extremely rare and of inadequate spatial resolution. Without direct measurement of the turbulent stresses in the surf zone, modeling of the viscous terms in the momentum balance is largely speculative. As these terms are critical in determining the vertical structure of the flow and the resulting bottom stresses, modeling efforts necessarily must be restricted. Nonetheless, existing field data and theory can be brought together to begin to constrain modeling 
efforts. We present herein a simplified model for mean cross-shore flows. The model predictions will be evaluated with data that, while limited in comparison to the ideal proposed above, provide constraint and verification consistent with the model. This avenue is explored in hopes that we may develop a useful first-order model suitable for the investigation of sediment transport by mean cross-shore flows. Success requires development of a model, with few free parameters, applicable to field situations where data is limited. This is only an initial step; results will support the contention [Svendsen et al., 1987] that more and better field data are still needed.

In the following, we will develop a simplified waveinduced mean flow model and compare it to field data from a barred surf zone. Modeling will encompass both the mean flow and the specifications of the wave field responsible for forcing of surf zone circulation. We will rely on published field-verified models of wave transformation as inputs to the mean flow model. We will attempt to indicate where field verification is lacking and where free parameters within the models are poorly constrained by data. This effort should point us to further model development and needed field observations.

\section{Model development}

Nearshore dynamics are generally expressed in the framework of the radiation stress tensor developed by Longuet-Higgins and Stewart [1964]. In this presentation we will discard the radiation stress formulation in lieu of a more classical form of the momentum equation. The formulation presented is, of course, completely equivalent to the radiation stress approach. The velocity model is based on solution of a simplified form of the momentum equation given by

$$
\left(u_{0}\right)_{t}+\left(u_{0}^{2}\right)_{x}+\left(u_{0} w_{0}\right)_{z}=-g\left(\eta_{0}\right)_{x}
$$

where $u_{0}$ is the cross-shore $(x)$ component of velocity (positive offshore), $w_{0}$ is the vertical (z) component of velocity, and $\eta_{0}$ is the water level. Here $u_{0}$ is equal to $u+u^{\prime}+U$, denoting the wave induced velocity, the turbulent fluctuations, and the time mean crossshore velocity, respectively. Similarly, $w_{0}=w+w^{\prime}$ and $\eta_{0}=\eta+\bar{\eta}$, where $\bar{\eta}$ is the time mean setup (setdown) of the sea surface associated with breaking (shoaling) waves (Figure 1). In this development, the mean flow is assumed to be the second-order response to forcing by the incident wave field. Equation (1) is strictly twodimensional, neglecting terms incorporating the alongshore velocity $v$ and alongshore gradients $\partial / \partial y$. These assumptions, along with assumptions that viscous terms and deviations from hydrostatic pressure are negligible, are appropriate for the time-averaged form of the equation to follow.

A number of terms will be neglected in this model, including the horizontal Reynold's stress term, $\boldsymbol{u}_{\boldsymbol{x}}^{\mathbf{2}}$. and nonlinear terms in $U$ (i.e., $\left.\left(U^{2}\right)_{x}\right)$. Neglecting the former term removes the primary mechanism of horizontal

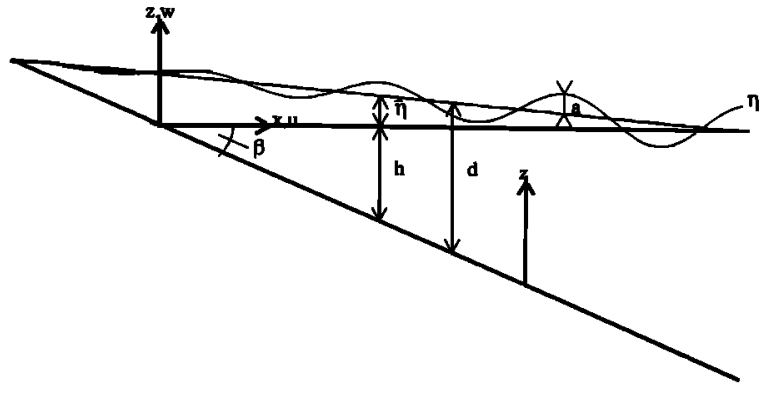

Figure 1. Coordinate frames and definitions for models and data.

mixing, and the resulting model is thus a strict local balance. Mixing terms are likely to be important where gradients in the wave-induced forcing are sharp. Previous studies of alongshore current dynamics have shown that horizontal mixing terms may be neglected when the wave-induced forcing is modeled as resulting from a random wave field [Battjes, 1972; Collins, 1974]. This is the path we shall follow in subsequent sections. As $U$ is assumed to be second-order relative to $u$, we may neglect the nonlinear terms in $U$. Nonetheless, gradients in $U$ may be large in the inner surf zone where the flow "overturns." Incorporating the nonlinear terms results in an intractable model, so they are neglected. The time-averaged form of equation (1) is then

$$
{\overline{\left(u^{2}\right)_{x}}}+\overline{(u w)}_{z}+{\overline{\left(u^{\prime} w^{\prime}\right)_{z}}}_{=-g} \bar{\eta}_{x}
$$

where $\overline{(~)}$ denotes time averaging. The remaining Reynold's stress term, $\overline{\left(u^{\prime} w^{\prime}\right)_{z}}$, is equated to an eddy viscosity formulation incorporating the mean shear. The resulting constituent equation for the mean flow field is

$$
{\overline{\left(u^{2}\right)_{x}}}+\overline{(u w)}_{z}-A_{y}\left(I_{z z}=-g \bar{\eta}_{x} .\right.
$$

$A_{v}$ in equation (3) is the eddy viscosity and is independent of $z$ and time. The above equation, integrated twice in the vertical, yields the mean current model to be investigated herein,

$$
\begin{aligned}
\iint \overline{\left(u^{2}\right)_{x}} \partial z^{\prime} & +\int \overline{(u w)} \partial z^{\prime}-A_{v} U \\
& =-g \bar{\eta}_{x} \frac{{z^{\prime}}^{2}}{2}+P z^{\prime}+Q
\end{aligned}
$$

where $(P, Q)$ are constants of integration. Here $z^{\prime}$ is a vertical coordinate, equal to $z+h$, extending from $z^{\prime}=0$ at the bed to $z^{\prime}=\eta+h$ at the free surface (Figure 1).

\section{Mean Current Model}

The model described by equation (4) provides a solution for the vertically varying mean current. Calculation of the current requires specification of the waveinduced flows, the vertical eddy viscosity, and the setup.

The wave velocities in equation (4) are given by linear wave theory. The horizontal wave velocity is 


$$
\begin{aligned}
u & =\frac{a \sigma}{\sinh (k h)} \cos (k x+\sigma t) \\
u & =A \cos (k x+\sigma t),
\end{aligned}
$$

where $a$ is the wave amplitude, $k$ the wave number, and $\sigma$ the frequency. The inclusion of a depth dependence to the wave velocity results in negligible change in the final solution over the parameter space defined by the data. The use of linear wave theory in a fully developed surf zone rather than a propagating bore theory is justified by the results of Guza and Thornton [1980] which showed that linear wave theory holds quite well within the surf zone.

Vertically varying mean flows result from the vertical structure of the wave-associated terms in the momentum equation. We will model this vertical variation as resulting from the presence of a turbulent boundary layer at the bed. Derivation of the vertically varying velocities follows closely the work of Russell and Osorio [1967], is described in Appendix 1, and results in a cross-shore velocity given by

$$
u=A\left[\cos (k x+\sigma t)-e^{-\phi} \cos (k x+\sigma t-\phi)\right],
$$

where $\delta=\left(2 A_{v} / \sigma\right)^{1 / 2}$ and $\phi=z^{\prime} / \delta$.

In the derivation of the wave-induced velocities (equations (6) and (20)) we have maintained the terms arising from the horizontal gradients in the wave amplitude and the bottom level. The contribution to the vertical velocity $-A \delta_{x}$ results from a horizontally varying eddy viscosity. This term will be neglected in subsequent model fitting to data. Results will show that while $A_{v}$ appears to vary across the surf zone, the term $A \delta_{x}$ is small. Equations (6) and (20) allow the determination of $U$ in equation (4). The boundary conditions for $Q$ and $P$ in equation (4) are given by

$$
\begin{aligned}
& 0=\left.U\right|_{z^{\prime}=0} \\
& 0=A_{v} \int_{0}^{d} U \partial z^{\prime}+A_{v}\left\langle\left.\eta u\right|_{d},\right.
\end{aligned}
$$

respectively. The boundary conditions imposed are a no-slip condition at the bed and continuity of mass flux across a vertical plane. The second term in the continuity condition, $\langle\eta u\rangle_{\left.\right|_{d}}$, approximates the mass flux above the mean water level. The mean current solution does not explicitly require imposition of a stress condition. We will incorporate an integral stress condition in the development of the wave-induced setup model which follows.

This model, with associated boundary conditions is essentially the same, with small deviations, as that proposed by previous investigators [e.g., Svendsen et al., 1987]. Again, we have chosen to model the eddy viscosity $A_{v}$ as independent of the vertical coordinate. Hansen and Svendsen [1984] drew attention to the importance of the much lower turbulence levels in the wave-induced bottom boundary layer. Svendsen et al. [1987] show that within this boundary layer, the eddy viscosity is small relative to that produced by wave breaking. They propose a two layer eddy viscosity model with constant $A_{v}$ within each of the two layers representing the boundary layer and the interior "undertow" flow. Their results show that the undertow velocities and shear stresses above the boundary layer are "remarkably insensitive" to the details of the boundary layer flow [Svendsen et al., 1987, pp. 11,846]. As our data are restricted to the fluid interior, we choose to use a $z$-independent model for $A_{v}$.

Deigaard et al. [1991], using the data of Svendsen et al. [1987] and Okayasu et al. [1988], develop a turbulence model resulting in a strongly $z$-dependent form of $A_{v}$. The lab data show strong near-bed mean shears with mean flow maxima quite near the bed. As we shall describe, the data discussed here differ substantially from the available lab data, having flow maxima much higher in the water column. Thus we feel application of laboratory-based models for $A_{v}$ is unwarranted and introduces additional degrees of freedom into the model where data are limited.

Svendsen et al. [1987] present a solution based on nonzero bottom velocity, $U_{b}$, rather than the no-slip condition proposed here. Again, they found the interior solution was insensitive to the choice of $U_{b}$. Given the restrictions imposed by the available data, we choose to avoid the introduction of an additional free parameter, $U_{b}$.

Stive and Wind [1986] avoided specifying a bottom boundary condition by prescribing a shear stress at the wave trough level. For purposes of sediment transport modeling, we choose to constrain the near-bed flow, which is most critical for sediment transport. It is widely acknowledged [Stive and Wind, 1986] that breaking waves result in an enhanced stress at the surface. This inadequacy in the model, largely related to an unknown eddy viscosity form, is a fundamental unknown in modeling efforts.

We have chosen the simplest models for $A_{v}$ and the bottom boundary condition. We feel these choices incorporate the essential physics at lowest order. Neither choice is strictly correct. Certainly, this model is inappropriate for describing accurately the stress at the bed and the near-bed boundary layer flow. We feel that more complicated models would be poorly constrained by the available field data.

The resulting form for the time mean velocity as a function of $(x, z)$ is given by

$$
\begin{gathered}
A_{v} U=\left(\frac{A^{2}}{2}\right)_{x} \frac{\delta^{2}}{4}\left[\phi^{2}-\frac{3}{2}+\phi e^{-\phi}(\sin \phi-\cos \phi)\right. \\
\left.+4 e^{-\phi} \sin \phi+e^{-\phi} \cos \phi+\frac{e^{-2 \phi}}{2}-\frac{2}{3} \frac{d z^{\prime}}{\delta^{2}}+3 \frac{z^{\prime}}{d}-\frac{13}{2} \frac{\delta z^{\prime}}{d^{2}}\right] \\
+A^{2} k \frac{\delta^{2}}{4}\left[-\frac{3}{2}+\phi e^{-\phi}(\sin \phi+\cos \phi)+2 e^{-\phi} \cos \phi\right. \\
\left.-e^{-\phi} \sin \phi-\frac{e^{-3 \phi}}{2}+3 \frac{z^{\prime}}{d}-\frac{3}{2} \frac{\delta z^{\prime}}{d^{2}}\right] \\
-A^{2} \delta_{x} \frac{\delta}{4}\left[1-\phi e^{-\phi}(\sin \phi-\cos \phi)-2 e^{-\phi} \sin \phi\right. \\
\left.-e^{-\phi} \cos \phi-2 \frac{z^{\prime}}{d}+4 \frac{\delta z^{\prime}}{d^{2}}\right] \\
+g \bar{\eta}_{x}\left(\frac{z^{\prime 2}}{2}-\frac{d z^{\prime}}{3}\right)-2 A_{v} \frac{z^{\prime}}{d^{2}}\left\langle\left.\eta u\right|_{d^{\prime}}\right.
\end{gathered}
$$


The above equation provides a solution for the mean currents generated by incident waves.

The first term in equation (8) is the radiation stress term describing the momentum flux gradients due to wave breaking. The second term represents the boundary layer streaming described by Longuet-Higgins [1953]. The third term, as noted, results from the horizontal variation in the eddy viscosity. This term will be neglected in further modeling. The fourth term represents the forcing due to the wave induced setup, and the final term is the continuity term reflecting the mass transport above the mean water level.

$A_{v}$ will remain a free parameter in the model to be determined by fitting to the observations. The magnitude of $A_{v}$ determined, representing the turbulent stresses generated by breaking waves, will control the modeled flow magnitude and vertical shear. As noted by reviewers, this is very similar to an alternative choice of the shear stress at an interior level as the free parameter. As $A_{v}$ is vertically uniform, the elevated turbulence levels associated with breaking waves will extend across the entire water column. This will result in near-bed turbulence levels which are greater than those associated with boundary layer processes in the absence of wave breaking. Thus the externally imposed turbulence due to breaking may be expected to extend the influence of the bottom boundary well into the water column. In order to calculate a solution for $U$ we need to specify the form for the time mean wave-induced setup, which is a necessary input to both the mean current model and the wave transformation model (to be described).

\section{Setup Model}

The mean elevation of the sea surface is modified by the presence of waves. The setup gradient is an important forcing for the cross-shore mean flow. No data is available to verify setup models under field conditions. Thus we follow, in a manner consistent with the velocity model, the formulation of Bowen et al. [1968], which has been verified under laboratory conditions.

Modeling the time mean setup, $\bar{\eta}$, requires a depthaveraged form of the momentum equation. Again discarding the horizontal Reynold's stresses and nonlinear terms in $U$, we vertically average equation (1). The vertical integral is approximated by

$$
\int_{-h}^{\eta_{0}}[] \partial z=\int_{-h}^{\bar{\eta}}[] \partial z+(\eta-\bar{\eta})[]_{\bar{\eta}} .
$$

Setting $\langle[]\rangle=\frac{1}{d} \int_{-h}^{\bar{\eta}}[] \partial z$, where $d=\bar{\eta}+h$, gives

$$
\begin{aligned}
{\left[d\left(u^{2}\right)\right]_{x} } & -h_{x}\left[u^{2}\right]_{\left.\right|_{-h}}-\frac{\partial}{\partial x}\left[\bar{\eta} u^{2}\right]_{\left.\right|_{\bar{\eta}}}+\eta\left[\left(u^{2}\right)_{x}\right]_{\left.\right|_{\bar{\eta}}} \\
& \left.+\left[u_{0} w_{0}\right]\right]_{\eta_{0}}-\left.\left[u_{0} w_{0}\right]\right|_{-h} \\
& =-g\left(\eta_{0}+h\right)\left(\eta_{0}\right)_{x}-[d\langle u\rangle]_{t} \\
& -\left.(\eta-\bar{\eta})\left(u_{t}\right)\right|_{\bar{\eta}}
\end{aligned}
$$

Equation (10) is further modified by noting that $w=$ $-u h_{x}$ at $z^{\prime}=0$, and $\left.u w\right|_{\eta_{0}}$ is approximately equal to $u \eta_{t}$ at $z=\bar{\eta}$. The above yield

$$
\begin{aligned}
{\left[d\left\langle u^{2}\right\rangle\right]_{x} } & -\frac{\partial}{\partial x}\left[\bar{\eta} u^{2}\right]_{\left.\right|_{\bar{\eta}}}+\eta\left[\left(u^{2}\right)_{x}\right]_{\left.\right|_{\bar{\eta}}} \\
& +\left.\left[u^{\prime} w^{\prime}\right]\right|_{\eta_{0}}-\left.\left[u^{\prime} w^{\prime}\right]\right|_{-h} \\
& =-g\left(\eta_{0}+h\right)\left(\eta_{0}\right)_{x}-[d(u)]_{t} \\
& -\frac{\partial}{\partial t}(\eta u)_{\left.\right|_{\bar{\eta}}}+\left.\bar{\eta}\left(u_{t}\right)\right|_{\bar{\eta}} .
\end{aligned}
$$

Time-averaging equation (11) gives the constituent form of the setup equation

$$
\begin{aligned}
{\left[\overline{d\left\langle u^{2}\right\rangle}\right]_{x} } & -\left[\left(\bar{\eta} \overline{u^{2}}\right)_{x}\right]_{\mid \bar{\eta}}+\left.\overline{\eta\left[\left(u^{2}\right)_{x}\right]}\right|_{\bar{\eta}}+\left.\overline{u^{\prime} w^{\prime}}\right|_{\eta_{0}}-\left.\overline{u^{\prime} w^{\prime}}\right|_{-h} \\
& =-g\left(\frac{\eta^{2}}{2}\right)_{x}-g d \bar{\eta}_{x}
\end{aligned}
$$

Equation (12) gives the general form of our solution. The turbulent stresses, $\overline{u^{\prime} w^{\prime}}$, are equated to the energy loss from the wave field, $1 / 2 g\left(a^{2}\right)_{x}$. The terms in (12) are calculated from the depth independent velocity (5). In this treatment we have neglected the details of the bottom boundary, because most of the dissipation occurs outside that region. The stress condition applied here does not incorporate a contribution due to the mean cross-shore flow, which is not a priori known. This is inconsistent with the no-slip condition applied in the mean current solution. While a more complicated iterative treatment of the stress is possible, this is inappropriate given the simplified form of $A_{v}$ chosen and the presumed dominance of the wave-induced stresses. Further discussion will show that, while the setup driven flow dominates the mean current solution, the general conclusions reached are insensitive to the setup model applied. Our setup solution is thus given by

$$
\bar{\eta}_{x}=-\frac{h}{g d}\left(\frac{A^{2}}{2}\right)_{x}-\frac{1}{g d}\left(\frac{A^{2}}{2}\right) h_{x}-\frac{3}{4 d}\left(a^{2}\right)_{x} .
$$

This formulation differs from that of Bowen et al. [1968] in three respects. First, it includes the term arising from the turbulent stresses. Second, it includes a term of the form $\left.\bar{\eta}_{x}\left[\overline{u^{2}}\right]\right|_{\bar{\eta}^{-}}$. A. Bowen (personal communication, 1992) has identified this term as arising from the dynamic effect of the setup, resulting in an additional pressure on the bed. Finally, Bowen et al. [1968] applied the simple surf zone parameterization, $H=\gamma h$, to reduce their equation to a linear function of the local bed slope. We will utilize a more complete wave transformation model to describe the local forcing. Comparisons of our model and Bowen et al. [1968], recast in terms of the wave transformation model, were carried out. The two models display virtually identical cross-shore behavior, with our model describing a slightly larger magnitude to the setup. As will be shown, the setup term is a critical forcing term for the generation of mean flows. Lack of field verification of setup models is a clear weakness in modeling efforts within the surf zone. 


\section{Experiment}

The data herein discussed resulted from an October 1982 field experiment at the Field Research Facility (FRF) of the U.S. Army Corps of Engineers' Waterways Experiment Station in Duck, North Carolina. The FRF is located on a long straight beach of a barrier island. A single, well-developed, shore-parallel bar was present throughout the data collection period.

The data discussed in this investigation were collected with the U.S. Geological Survey sled system, which consists of an instrumented sled towed along the bed, both offshore and onshore, with a doubledrum winch and triangular line arrangement [Sallenger et al., 1983]. On the sled, 4-cm-diameter electromagnetic current meters (Marsh-McBirney model 512) were mounted in a vertical array at $0.5,1.0$, and $1.75 \mathrm{~m}$ above the bed. Also mounted on the sled was a pressure sensor used to measure local depths. Data were telemetered to a shore receiving station where they were digitally recorded at a rate of $2 \mathrm{~Hz}$. All record lengths were $34.1 \mathrm{~min}$. As the sled moved along the shore-normal transect, the nearshore profile was measured using an infrared range finder on the beach, sighting on optical prisms mounted on top of the sled's mast. The sled system was set up $500 \mathrm{~m}$ north of the FRF pier to avoid the effects the pier has on local flows and sediment trans port.

Mounting the current meters on a movable platform has both advantages and drawbacks. The primary advantage is the increased horizontal resolution allowed by a mobile instrument platform. Two significant drawbacks have been noted. First, the movement of the instrument platform results in nonsynoptic coverage. While we believe the wave field was relatively stationary over the course of a single sled transect, tidal levels fluctuated significantly. Second, the sled provides poor control over current meter orientation. Vigourous alongshore currents certainly skewed the sled from a shore-normal orientation. A digital compass mounted on the sled failed to provide reliable orientation data. We have discarded those data runs where sled orientation appears to be unreliable. In order to minimize flow disturbance, the current meters were cantilevered away from the sled. The current meter closest to the bed was $1.5 \mathrm{~m}$ upstream of the sled, into the alongshore current. This should minimize flow disturbance effects but the potential for turbulence generation by flow around the sled cannot be dismissed.

While the vertical resolution provided by 3 current meters is somewhat limited, the cross-shore coverage provides a rich data set. Conditions observed included strong mean currents, a necessary condition for use of Marsh-McBirney current meters, which require mean flows in excess of approximately $5 \mathrm{~cm} \mathrm{~s}^{-1}$ for reliable measurement of the mean in a vigourous surf zone. Shepard and LaFond [1939] have suggested that energetic two-dimensional circulation may become unstable to three-dimensional perturbations and break up into a horizontal circulation associated with rip currents. No three-dimensional flows were noted in the field.

\section{Data}

The data discussed here were obtained during a 3day storm spanning October 10-12, 1982. During this period of high energy, the wave period, steepness, and spectral width of the incident spectrum varied considerably (Figure 2a). On October 10, strong northeast winds, which reached $13 \mathrm{~ms}^{-1}$, generated steep waves with a broad incident spectrum. The observed spectra for October 10 (Figure 2b) were highly variable, clearly documenting the buildup of storm conditions. By October 12 the storm had moved offshore and local winds were light; waves were principally of the swell type and, relative to October 10 , the incident spectrum had significantly decreased in width. Throughout the 3-day period, the sled transects were within the inner $50 \%$ of the surf zone. On October 12 , significant breaker heights of nearly $4 \mathrm{~m}$ resulted in a surf zone width of roughly $900 \mathrm{~m}$, and the breaker zone occured $600 \mathrm{~m}$ seaward of the outermost data station. Spectra for October 11 and 12 (Figures $2 c$ and $2 d$ ) are much more stable than those of October 10, although some variation is noted.

The data for October 10 were, as previously mentioned, marked by a rapidly varying incident wave field. In addition, alongshore currents were vigourous, approaching $1 \mathrm{~ms}^{-1}$ at most of the observation stations. The data clearly suggest that the instumented sled was subject to significant rotation by these currents. As the absolute orientation of the waves is unknown, the data from October 10 is ignored in the subsequent discussion of mean flows.

In the following the mean flows are calculated over the entire 34.1 min of each data run. Incident wave heights are calculated from the velocity spectra across the frequency range 0.05 to $0.33 \mathrm{~Hz}$, thus removing the effects of infragravity and turbulent motions respectively. While the upper cutoff is somewhat arbitrary, results are insensitive to the particular choice of the cutoff frequency.

Observed mean currents for October 11 and 12 are energetic and exhibit a classic "undertow" form (Figures 3 and 4). The bulk of the mean flows are offshore directed. Onshore-directed flows are observed high in the water column at the inshore stations. On both days, maximum velocities are observed well above the bed, in contrast to the laboratory data discussed by Deigaard et al. [1991]. The lab data show a strong near-bed shear and flow maxima within the bottom $30 \%$ of the water column. This leads us to question whether the stress distribution in laboratory experiments is generally applicable to field conditions. Run numbers are annotated in Figures 3 and 4 . Numbers indicate the sequential order in which the stations were occupied. Water depths for the individual runs are indicated by the height of the vertical "mast." The profiles shown in Figures 3 and 4 are the daily profiles taken prior to the data collection by the sled. There is a substantial offshore migration of the bar between October 11 and October 12 [Sallenger et al., 1985]. As an aside, the alongshore velocities for October 12 (Figure 4b) show 

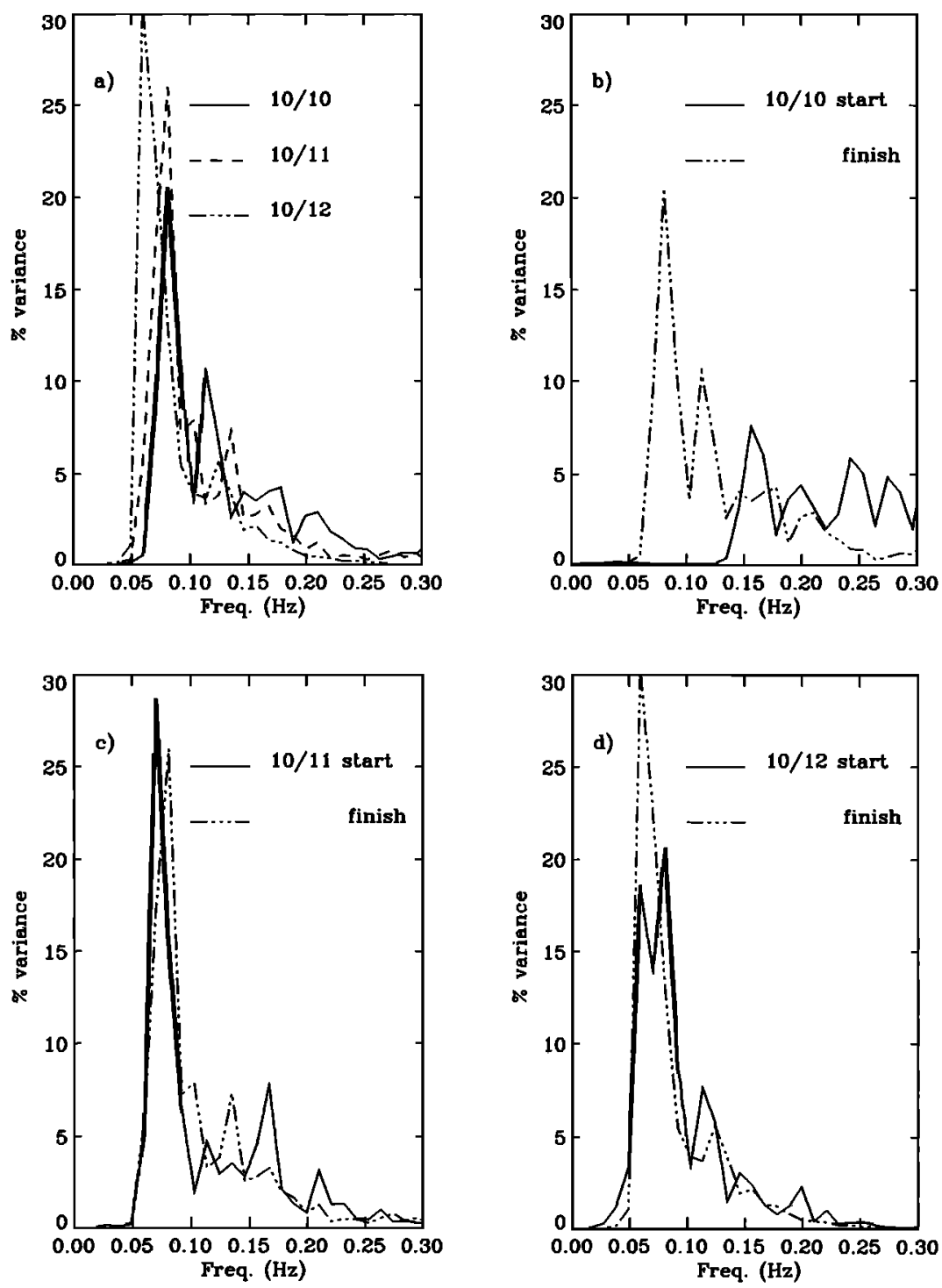

Figure 2. (a) Deepwater wave spectra from Field Research Facility wave rider. Wave spectra at the beginning and end of data collection for (b) October 10, (c) October 11, and (d) October (12).

a marked vertical shear. Simulianeous investigation of the alongshore and cross-shore flows may better constrain the flow models but, in the absence of reliable measures of incident wave angles, is not possible here.

The mean flow model requires specification of both the wave heights (observed) and the gradients due to breaking and shoaling. The wave transformation across the surf zone is calculated following Thornton and Guza [1983]. Their model for wave height and dissipation across the surf zone is based on a probabilistic breaking wave model. The model of Thornton and Guza [1983] has been shown to work quite well across simple topography. The wave transformation model has one free parameter, $B$, nominally the percentage of foam on the breaker face. $B$ is not a measurable parameter; nor is it widely known for a variety of conditions. For this case, $B$ will be fit to the observed wave heights recorded by the instrumented sled. The difficulty in estimating $B$ limits the model's reliability where adequate observa- tions for the determination of $B$ are unavailable. It is clear that a reliable parameterization of $B$ is a desirable aim for future studies.

A description of the shoaling wave model and its application is deferred to Appendix 2. Equation (28) (Appendix 2), describing the wave evolution, is solved for the values of $B$ that best model the observed values of $H_{r m s}$ for the 3 days. The solution is calculated across the profile beginning with an input condition of $H_{r m s}=\gamma h$ and $\bar{\eta}=0$ at a position offshore of the outermost data position. Because of the nonsynoptic: nature of the observations, each data run (i.e., each sled location) was separately modeled and a best-fit value of $B$ determined for each day. Figure 5 shows the results for all days. The solid line represents the wave heights given by the value of $B$ that provides the best overall fit for the day in question. Asterisks indicate the observed wave height at each sled location. Observed wave heights in Figure 5 have been normalized 

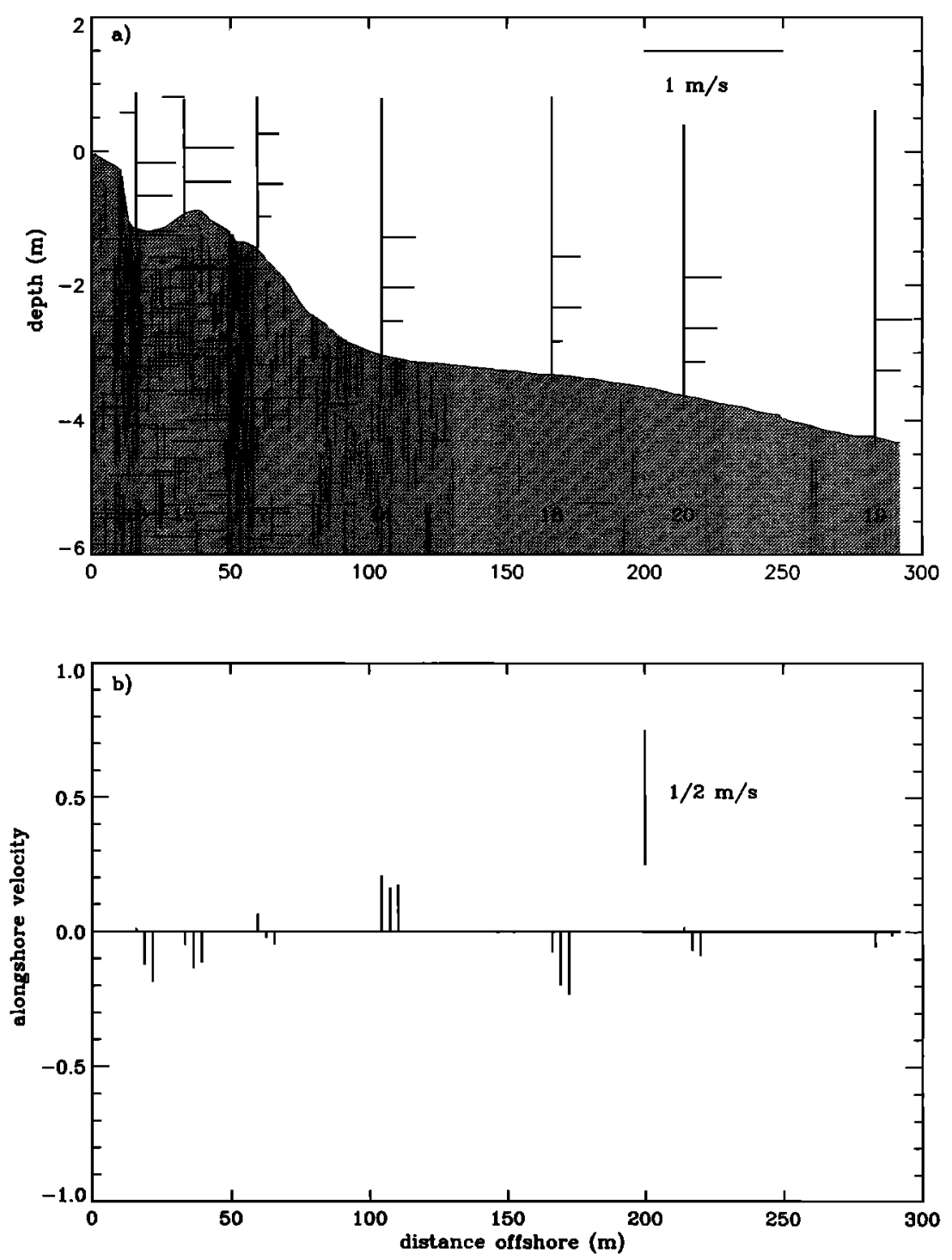

Figure 3. Velocity data for October 11. (a) Cross-shore mean velocities. Numbers beneath profile indicate data run. (b) Alongshore mean velocities; sensors increase in height above the bed going offshore.

to reflect the model error while providing a synoptic picture. The values underneath the model curve show the best-fit value of $B$ for each separate data run. For all days, $B$ increases shoreward across the surf zone, suggesting increasing dissipation in shallower water (for a given wave height). Inputs into the mean current model will be based on the value of $B$ determined to be best for the entire day's data. This choice was made, rather than using a separate value of $B$ for each run, to maintain the simplicity of the model. Although the variation in $B$ is marked, the wave heights predicted, assuming a constant value of $B$, are good estimators of the observed wave heights. Figure 5 also shows (dashed line) the predicted wave heights for $H_{r m s}=\gamma h$. As suggested by the regression in Figure 15 (Appendix 2) this simple model differs significantly from observations over the bar.

Figure 6 shows the regression of $B$ against the surf similarity parameter for this dala set and the data pre- sented in Thornton and Guza [1983]. The surf similarity parameter is given by

$$
\xi_{0}=\frac{\tan \beta}{\left(H_{0} / L_{0}\right)^{1 / 2}},
$$

where $H_{0}$ and $L_{0}$ are the deepwater wave height and wavelength respectively. The beach slope, $\beta$, is estimated by the rms slope across the profile for the Duck data modeled here. The results of the wave height modeling carried out here suggest that $B$, as it functions in the model of Thornton and Guza [1983], is not constant across the surf zone. Nonetheless, we have chosen a constant value of $B$ across the entire surf zone. Insofar as $B$ should be related to breaker type, the surf sinilarity parameter may be a plausible parameter to explore. What is clear is that $B$ varies by a factor of two across the limited parameter space slown. Such variation results in large changes in the predicted wave heights and dissipation across the surf zone. As $B$ significantly im- 

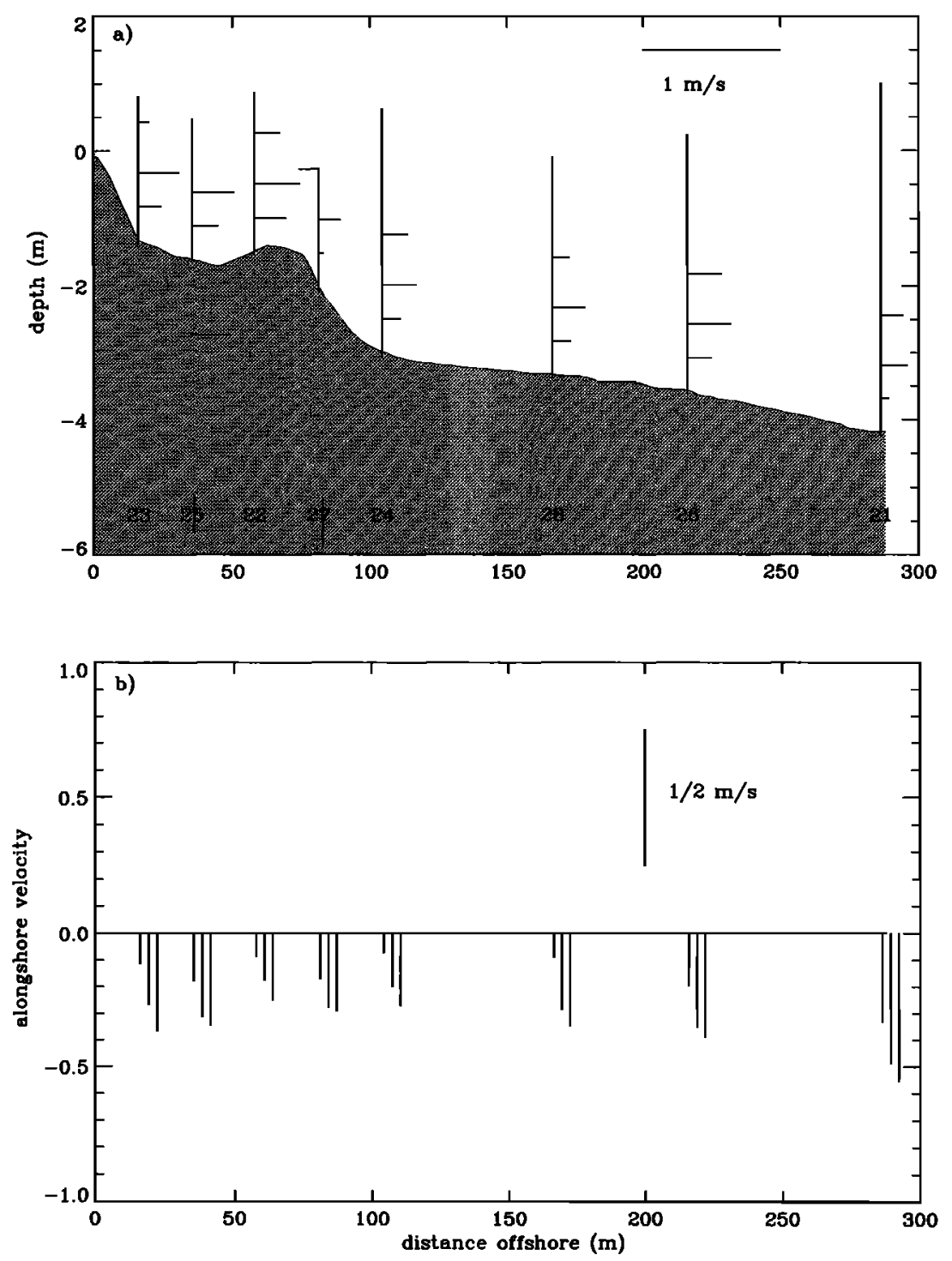

Figure 4. Velocity data for October 12. (a) Cross-shore mean velocities. Numbers beneath profile indicate data run. (b) Alongshore mean velocities: sensors increase in height above the bed going offshore.

pacts prediction of the breaking wave transformation, it may be viewed as an important parameter in subsequent model fitting. The sensitivity of the model results to variability in the input wave heights will be addressed in a subsequent section.

\section{Results}

Utilizing the results of the wave transformation model velocity profiles were calculated for each sled station from October 11 and 12. At each station, $A_{v}$ was determined as that value which yielded the best least squares fit to the three observed velocities. This yielded a clearly superior fit of model to data in contrast to choosing a constant $A_{v}$ across the surf zone.

The resulting model fits are shown in Figure 7. The model clearly reproduces the "undertow" characteristics of the flow, with interior flows directed offshore. It must be noted that the model fits presented for stations 17 and 27 are misleading. The value of $A_{v}$ required to produce the fits shown are extremely large relative to adjacent stations. The value of $A_{v}$ necessary to fit the observed flow is at least a factor of 2 greater than any adjacent location. As both stations are located on the bar flank, this may reflect the energetic conditions prevailing as waves enter rapidly sloaling depths. In both cases the model substantially overpredicts velocities in the lower portion of the water column. In this region of sharp gradients the local balance approach of the model may be inappropriate. An alternative explanation may derive from flow disturbance by the upstream bar. The near bed velocity is particularly reduced at station 27 , occupied during low tide conditions. In this case the bar, occupying a significant fraction of the water depth, may significantly disturb the flow.

Extremely strong onshore currents with large shears are predicted near the free surface. Where near-surface 

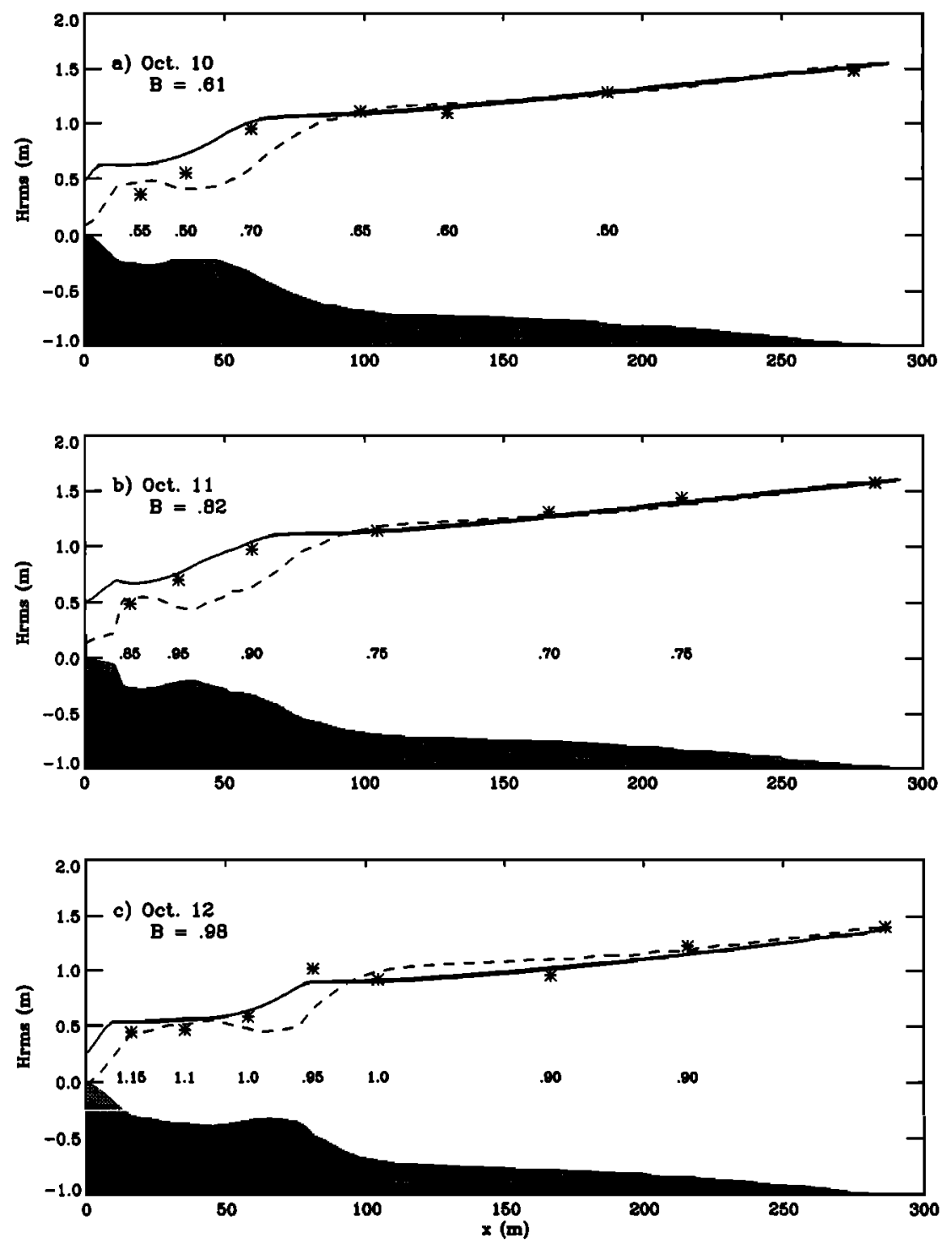

Figure 5. $H_{r m s}$ observed (asterisk) and resulting from Thornton and Guza, [1983] model solution. Solid line is model solution, dashed line is $H_{r m s}=\gamma h$. Values beneath model curve give value of $B$ which results in best prediction for each data run.

observations are available, onshore flows consistent with the predictions are seen. The data, with limited observations near the free surface, do not adequately constrain the model in this region. Observations in this region are critical to investigating possible alternative models for the eddy viscosity.

Figure 8a shows the complete data set. In general, the observed velocities are well reproduced by those predicted by the model. Figure $8 \mathrm{~b}$ illustrates some of the systematic failures of the model. The error (observed minus predicted) is plotted against the nondimensional depth for each sensor. In the center of the water column the model tends to overpredict the offshore flow. Near the bed the model tends to underpredict the offshore flow. The most plausible explanation for these failures is that the parameterization of $A_{v}$ as $z$ independent is incorrect. A reduced $A_{v}$ near the bed, as suggested by Svendsen et al. [1987], would allow a stronger near-bed shear to exist, thus resulting in larger near-bed flows.

\section{Discussion}

This model, in general, predicts a velocity profile marked by offshore flows over the lower portion of the water column with an offshore velocity maximum near the center of the water column. Onshore flows, marked by strong vertical shear, are predicted for the upper portion of the water column. The detailed flow structure is strongly controlled by the value of $A_{v}$ chosen. If $A_{v}$ is not a well defined parameter, this model is of limited, if any, usefulness. As previously noted, the model's failures may suggest that a depth-dependent form for the eddy viscosity is appropriate. Our data set, limited in vertical resolution, does not allow explicit investigation of this question. The fitted values of $A_{v}$ should reflect the horizontal structure of the eddy viscosity, especially since the bulk of the observations are well removed from the boundaries of the flow. 


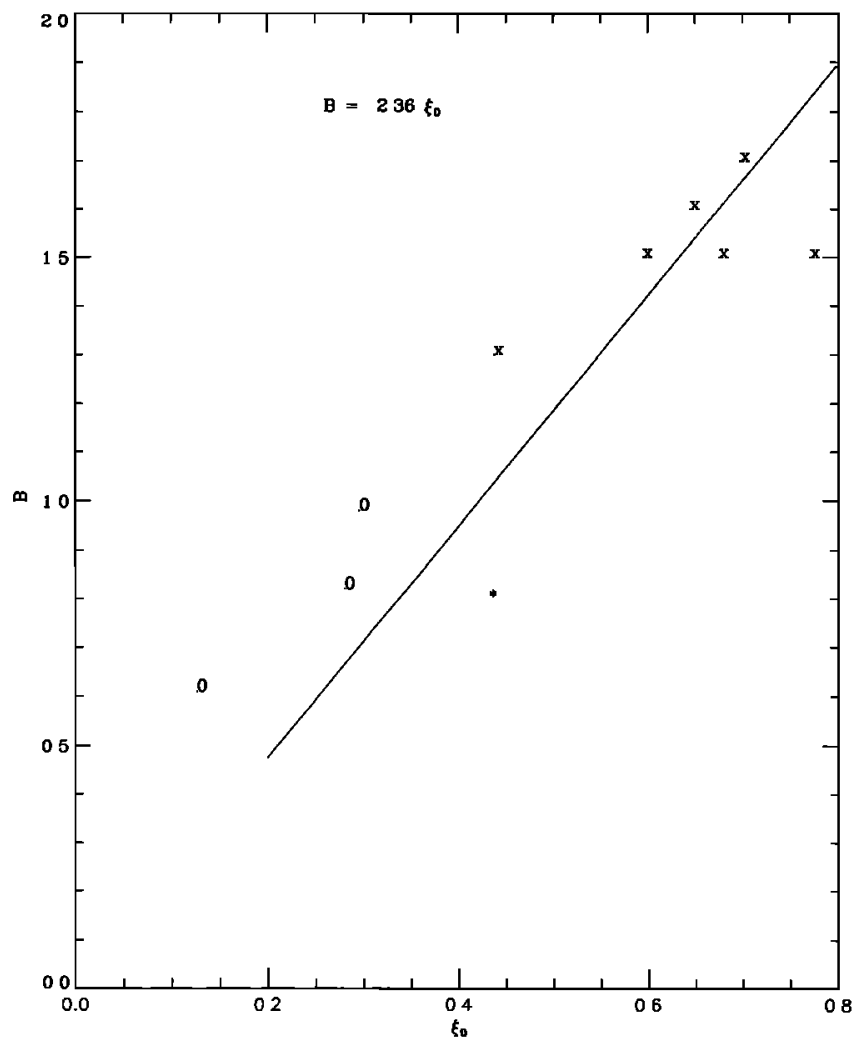

Figure 6. Regression of fitted $B$ against the surfsimilarity parameter. Included data are from Thornton and Guza, [1983] ( crosses (field data), and asterisks (lab data)) and this study (0).

Figure 9 shows the regression of $A_{v}$, as determined by the model fitting, against four "candidates" for parameterization. Figure $9 \mathrm{a}$ shows the regression of $A_{v}$ against $a^{2} \sigma$, a mixing parameter suggested by Thornton [1970]. In this discussion the two points from the outer flank of the bar (runs 17 and 27) have been discarded because they resulted in values of $A_{v}$ which clearly deviate from any trend. 'The parameter $a^{2} \sigma$ provides some predictive skill across the factor of 6 range observed. Predictive skill is somewhat increased by the choice of $a k d\left(\epsilon_{b} / \rho\right)^{1 / 3}$, incorporating the bore dissipation from the model of Thornton and Guza [1983], as the independent variable of the regression. Physically, it seems clear that $A_{v}$, representing turbulent fluctuations associated with wave breaking, should be related to $\epsilon_{b}$. As previously discussed, $A_{v}$ may be expected to control the internal shear stress, which has been shown to be strongly related to dissipation [ $D e-$ Vriend and Stive, 1987]. Parameters incorporating $\epsilon_{b}$ have been chosen as those dimensionally consistent parameters which best describe the variability in fitted values of $A_{v}$. Marginally greater skill is exhibited by regression of $A_{v}$ versus $(d k)^{2} \sigma^{-1}\left(\epsilon_{b} / \rho\right)^{2 / 3}$. Both parameters, which are functions of $\epsilon_{b}$, have behavior which is largely depth controlled, suggesting that mixing length arguments may be important.

The final parameter investigated is $d \sqrt{g d}$, a parameter suggested by the lab data of' Svendsen et al. [1987] and Stive and Wind [1986]. Predictive skill is lowest for this choice. More importantly, the regression slope is an order of magnitude lower than that found by Svendsen et al. [1987]. This again suggests that laboratory investigations of wave-induced stresses may be of limited application to field conditions.

Some additional points may be highlighted by further examination of the regression plots. Stations 19 and 21 display the highest fitted values of $A_{v}$. These points represent the offshore limits of the data collection on the two days. To assure that these points are not controlling the fitted relation, a separate regression was calculated after removal of station 21. Regression results are shown by the dotted line and by the regression parameters within brackets in Figures $9 \mathrm{~b}$ and $9 \mathrm{c}$. The regression is not markedly impacted by removal of this point, and the fitted slope is not significantly changed. Stations 16 and 23 also consistently deviate from the fitted relations. These points both lie at the base of the steep inner beach face. This clustering of values from similar locations, along with the model's failure to describe bar flank observations, suggests that the data exhibit consistent behavior across the two days; and model shortcomings are similarly consistent.

The values of $A_{v}$ fitted vary by a factor of approximately 10 (from 0.0055 to $0.075 \mathrm{~m}^{2} \mathrm{~s}^{-1}$ ). Thus field measurements of turbulent stresses should resolve the
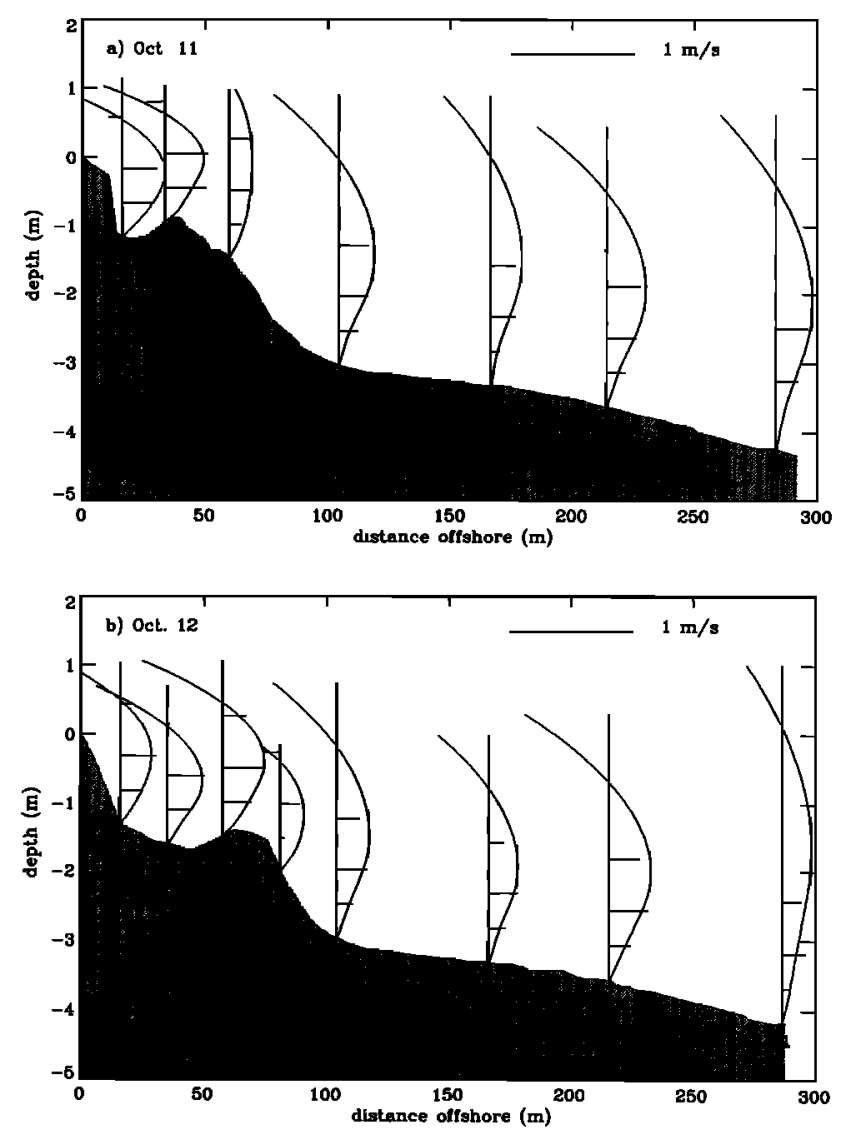

Figure 7. Best fit velocity $(U)$ solution for (a) October 11 and (b) October 12. $A_{v}$ chosen at each station to best reproduce the observations. 

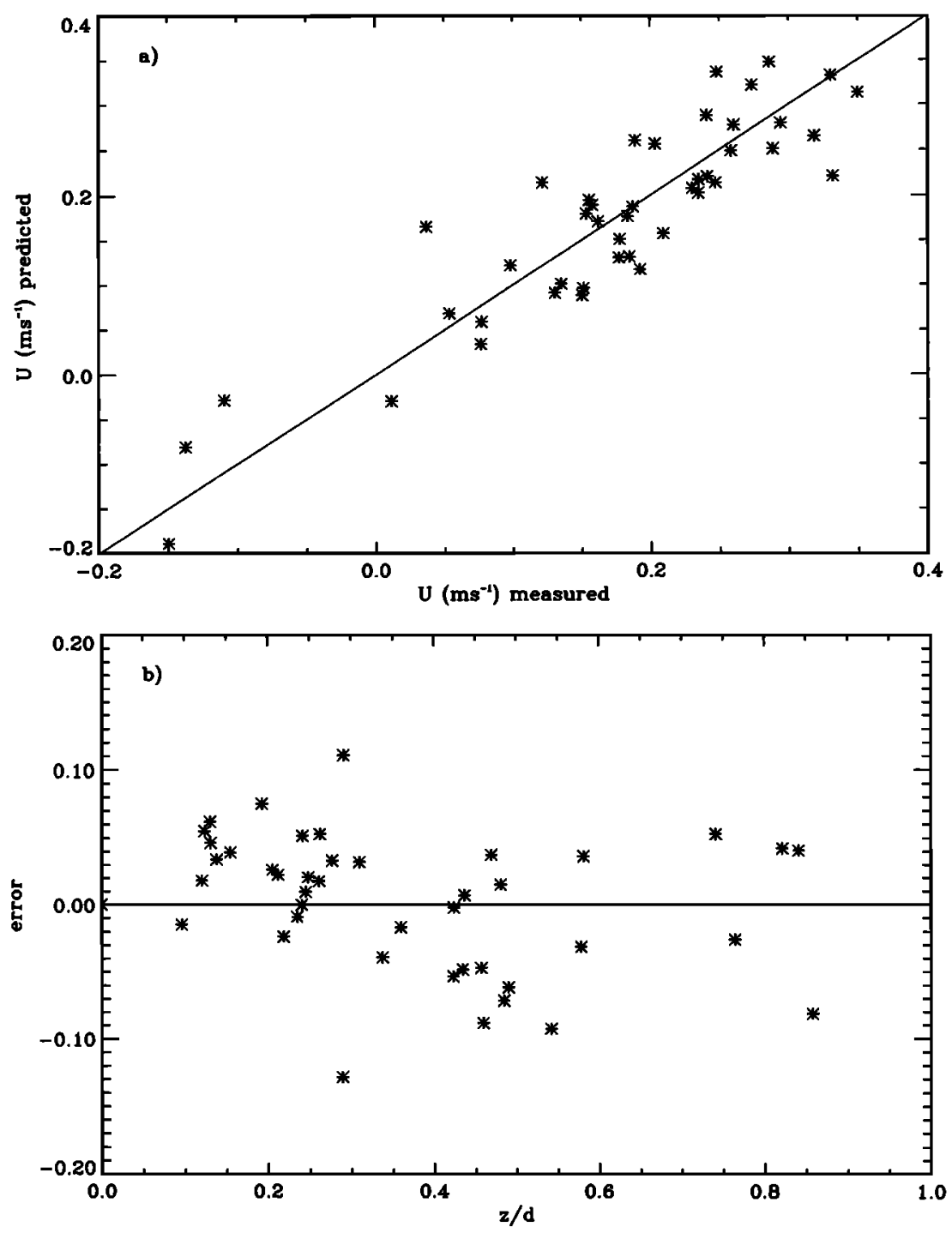

Figure 8. (a) Observed versus predicted velocity. (b) Error (observed minus predicted) versus normalized depth $z / d$.

observed variability if, in fact, the model predictions reflect real world variability.

The values of $A_{v}$ fitted are clearly dependent on the input parameters resulting from the wave transformation and setup modeling. The "error bars" in Figure 9 are an attempt to quantify the sensitivity of the results to the model inputs. The vertical error bars represent model results allowing $H_{r m s}, H_{x}$, and $\eta_{x}$ to vary by $\pm 20 \%$. The extreme values of $A_{v}$ resulting from all possible values of the input parameters are shown as error bars. Horizontal error bars in Figures $9 b$ and $9 c$ represent $a \pm 20 \%$ error in estimates of $\epsilon_{b}$. Clearly, the trends exhibited in Figure 9 remain significant. The predictive skill of the model is not greatly degraded given this level of variability in the input terms. Model fitting was also carried out using the setup model of Bowen et al. [1968]. The general functional form of $A_{v}$ was maintained. As will be shown later, the setup is the dominant forcing term for mean currents, and changes in the input setup gradient are largely balanced by relatively modest changes in $A_{v}$.
The implications of the parameterizations of $A_{v}$ as a function of $\epsilon_{b}^{n}$ are shown in Figures 10 and 11. These figures show the predicted currents across the bar for the the parameterization $A_{v}=f\left(\epsilon_{b}^{2 / 3}\right)$ and a constant $A_{v} \neq f(x)$ at varying tidal stages. These results differ only slightly from applying the parameterization $A_{v}=f\left(\epsilon_{b}^{1 / 3}\right)$. Figure 10 shows the results for a tidal stage $25 \mathrm{~cm}$ below MWL. The cross-shore variability in $A_{v}$ is shown in Figure 10c. The low values of $A_{v}(x)$ inshore of the bar allow strong vertical shears to exist in the current structure. In contrast, across the bar crest and offshore, the variable $A_{v}(x)$ greatly reduces the flow velocities compared to the constant $A_{v}$ case. While the data does not incorporate sufficient cases (low ticle or sensors near the bar crest) directly comparable to this case, the constant $A_{v}$ results are clearly unrealistic.

Figure 11 shows a high-tide scenario with still water levels of $1 \mathrm{~m}$ above MWL. Clearly, the values of $A_{v}$ are enhanced relative to the low-water scenario. The model results are similar to those shown previously, although the impact of allowing $A_{v}$ to vary is somewhat 

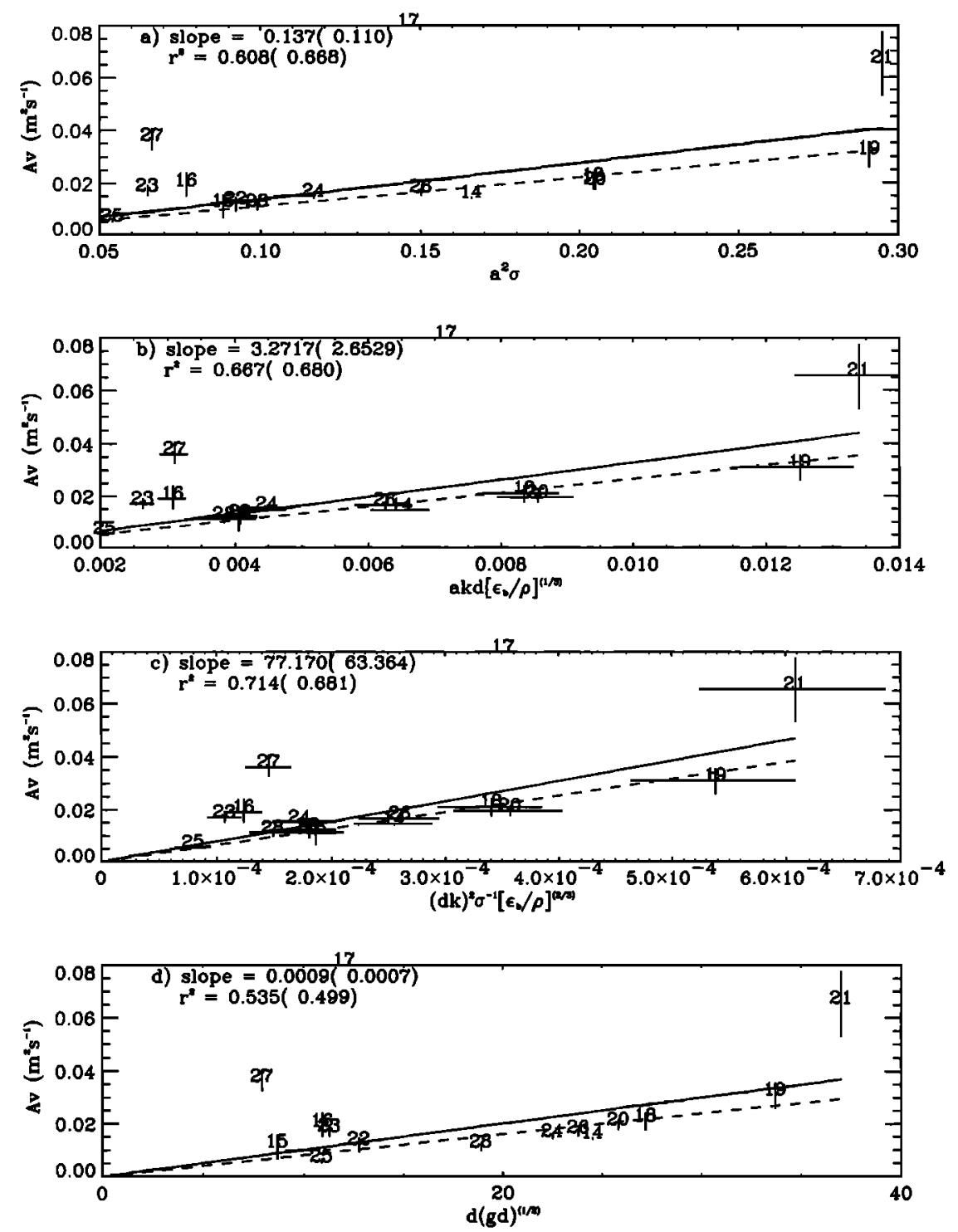

Figure 9. Regression of best fit $A_{v}$ against (a) $a^{2} \sigma$, (b) $a k d\left(\epsilon_{b} / \rho\right)^{1 / 3},(\mathrm{c})(d k)^{2} \sigma^{-1}\left(\epsilon_{b} / \rho\right)^{2 / 3}$, and (d) $d \sqrt{g}$. Error bars are described in text.

reduced. Investigation of nearshore stresses should confirm, or contradict, the predicted horizontal and tidal level variation. The elevated values of $A_{v}(x)$ seen across the bar in both scenarios may contribute to the reduced velocities observed on the outer bar flank.

The uniformly greater velocities and larger shears exhibited at lower water levels have significant implications for sediment transport modeling. The model suggests that tidal level may have a rather large influence on beach response to storm events. This model prediction is certainly amenable to evaluation by further data collection on natural beaches.

In none of the scenarios presented were near-bed onshore flows predicted. The reason for this result can be seen by examination of the dominant terms within the velocity model (equation (11)). The model prediction across the nearshore (for the high-tide scenario) is again shown in Figure 12. The "image" shown in Figure 12b identifies the dominant term contributing to the model solution. Contours show the ratio of the dominant term to the second largest term in magnitude (shown in Figure 12a, along with the underlying profile). Figure 12, and Figure 13 (the low-tide scenario), are virtually identical for either of the eddy viscosity parameterizations incorporating $\epsilon_{b}$. The left-hand side of Figure 13 (blacked out) represents a region which is subaerial at the specified tidal level.

It is clear that in both tidal scenarios, the modeled flow is dominated by the contribution from the mean setup gradient. Away from the bed $(z / d>0.4)$ the secondary input is primarily from the continuity term, an estimate of the mass transport above the trough level. The contour values suggest that across much of the water column, the setup term is a factor of 2 or more larger than the lesser contributors. Neither the setup term nor the mass transport term are well defined by observations. Further field work constraining these terms is clearly indicated. 

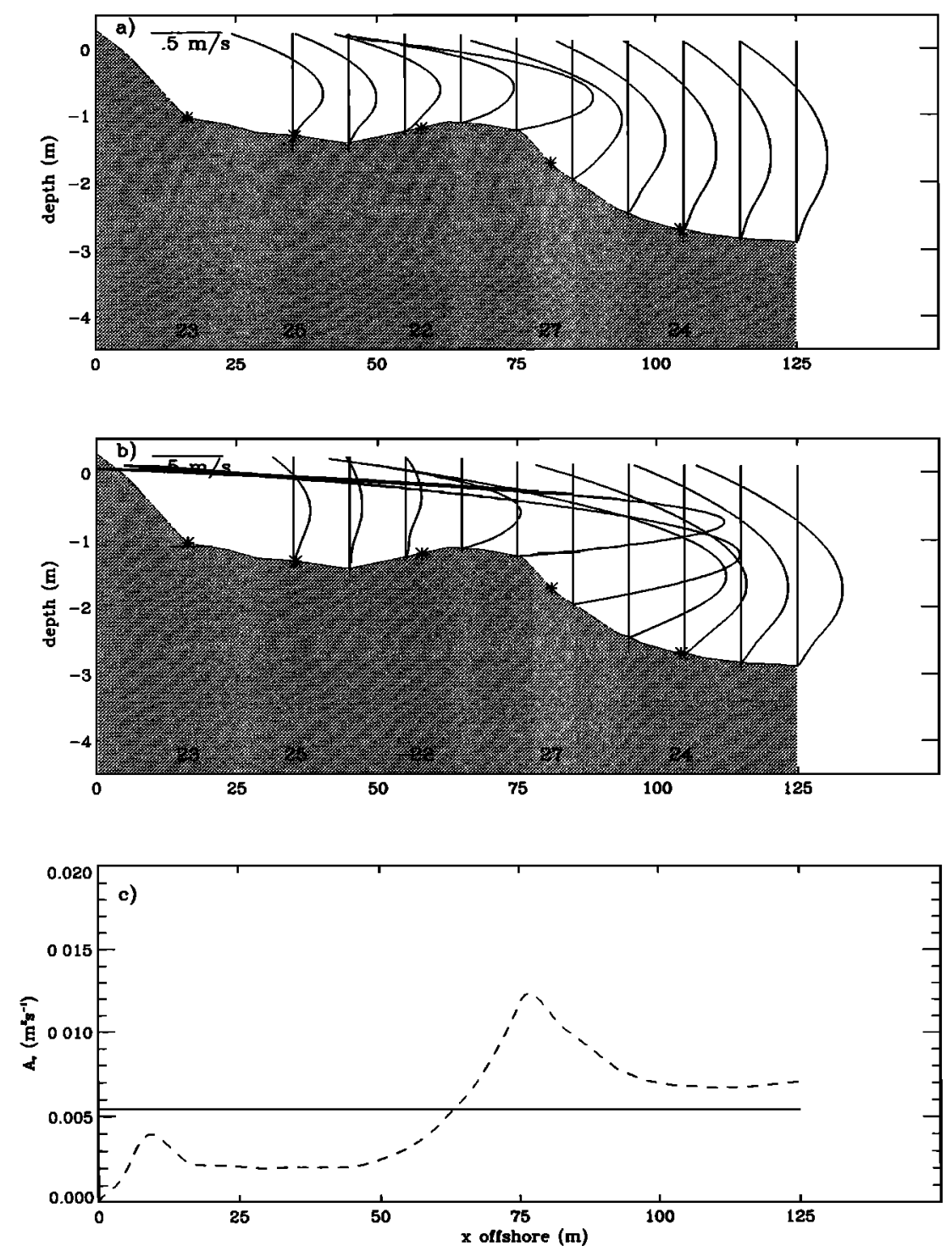

Figure 10. Model predicted flows across the bar for (a) $A_{v}=a k d\left(\epsilon_{b} / \rho\right)^{1 / 3}$ and (b) $A_{v}=$ $(d k)^{2} \sigma^{-1}\left(\epsilon_{b} / \rho\right)^{2 / 3}$ for low-tide conditions. (c) The spatial structure of $A_{v}\left(=f\left(\epsilon_{b} / \rho\right)^{1 / 3}\right)$ (dotted line).

Near the bed the secondary terms are primarily the boundary layer streaming term $\left(A^{2} k\right)$, with the radiation stress term $\left(\left(A^{2}\right)_{x}\right)$ encompassing significant regions of the low tide scenario. Negative contour values suggest that these terms are acting in a contrary sense to the setup term which dominates. Thus while the near-bed flow remains offshore, it is somewhat reduced by the influence of these secondary terms. A. Bowen (personal communication, 1992) has observed onshore near-bed flows over a barred nearshore. Our model uniformly predicts offyhore flows. Moderate variation of the input parameters $\left(H_{r m s}, H\right.$, and $\left.\eta_{x}\right)$ did not result in onshore flows. Further Inodel runs across a wider parameter space including $\beta$ and $\sigma$ may be warranted.

Figure 14 shows the contributing terms to the mean velocity (equation (11)) for the low-tide scenario. The right-hand panels show the cross-shore structure; Fig- ure $14 \mathrm{~b}$ also shows the underlying beach profile and sensor locations for October 12. The left-hand panels show the vertical structure for the the two stations indicated by the asterisks. Figure 14 shows that the wave induced forcing exhibits steep gradients across the bar and in the vicinity of the steep inshore beach face. As suggested by the previous figure, the setup term dominates the solution, uniformly forcing offshore flow in the lower portion of the water column. The continuity term, increasing rapidly toward the surface, becomes increasingly important high in the water column. The radiation stress term $\left(A^{2}\right)_{x}$ largely acts contrary to the setup term (as suggested by the contour values in Figure 13). While the boundary layer streaming term (Figure 14d) exhibits strong near bed gradients, the coefficient describing the horizontal variation is everywhere small. Thus the near-bed flows are everywhere offshore. 

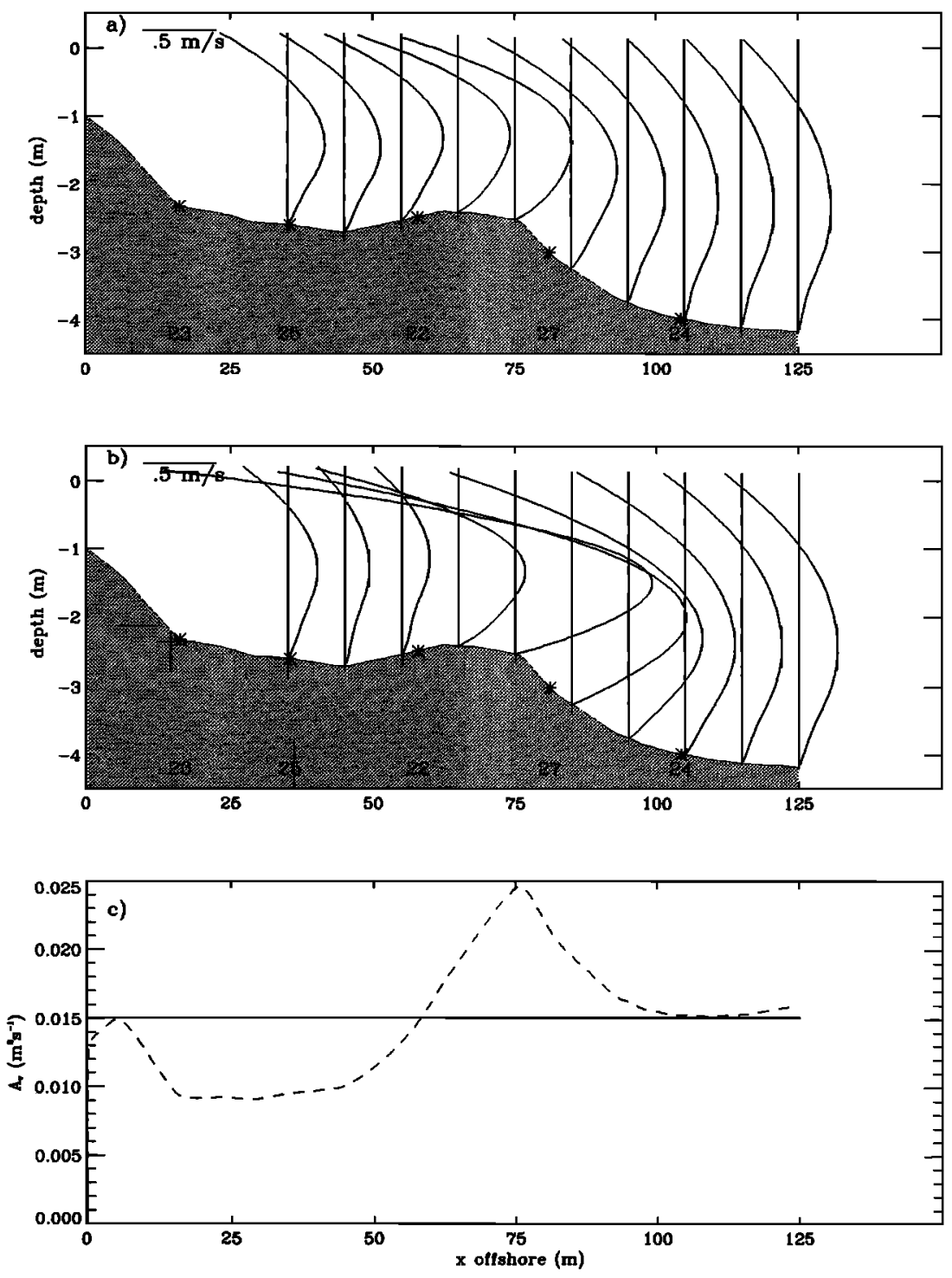

Figure 11. Model predicted flows across the bar for (a) $A_{v}=\operatorname{akd}\left(\epsilon_{b} / \rho\right)^{1 / 3}$ and (b) $A_{v}=$ $(d k)^{2} \sigma^{-1}\left(\epsilon_{b} / \rho\right)^{2 / 3}$ for high-tide conditions. (c) The spatial structure of $A_{v}\left(=f\left(\epsilon_{b} / \rho\right)^{1 / 3}\right)$ (dotted line).

The topmost panels in Figure 14 show the behavior of the term incorporating $\delta_{x}$. This term, as previously suggested, is everywhere small, validating its omission from the model fitting.

\section{Conclusions}

Overall, the model predicts the observed velocities quite well. Model results depend critically on the chosen value of $A_{v}$. The parameterizations presented, $A_{v}=f\left(\epsilon_{b}\right)$, describe the bulk of the data. Results clearly suggest that $A_{v}$ varies substantially across the surf zone. Deviations from these parameterizations show systematic behavior across the 2 days investigated. This may provide useful information in modifying the model and determining an improved parameterization of $A_{v}$. Model errors may suggest that a vertically vary- ing $A_{v}$ is appropriate, particularly near the boundaries. Obviously, improved observations of mean flows adjacent to the surface and bottom boundaries are needed, as are direct observations of turbulent stresses.

The predicted mean flows are one crucial component in development of a reliable model of sediment transport and beach evolution within the surf zone. Without simultaneous investigation of the wave-induced transport, it is difficult to evaluate the impact of any mean flow model. The mean flows predicted are consistent with the noted offshore migration of the bar [Sallenger et al., 1985]. Quantitative prediction of profile evoulution would require careful evaluation of the near-bed flows and stresses, a task inconsistent with the simple model we have presented.

The model depends heavily on knowledge of the incident wave field and the mean setup of the sea surface. 

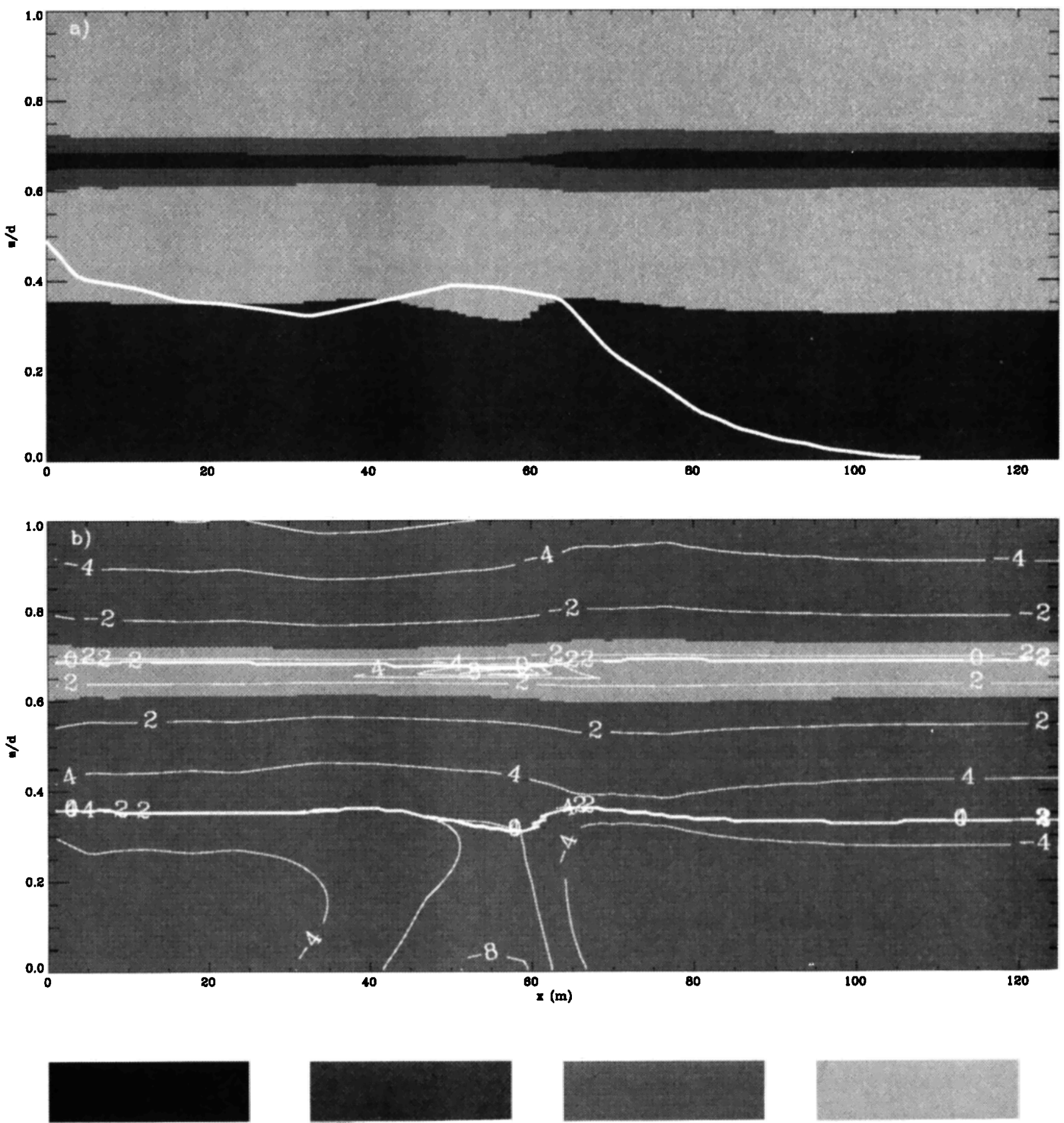

$\left(\frac{A^{\circ}}{2}\right) \times \frac{8^{\circ}}{4}$

$A^{2} k_{\frac{6}{4}}^{\frac{0}{4}}$

$\mathrm{g} \bar{\eta}_{\mathbf{x}} \mathrm{d}^{2}$

$-2 A_{v} \overline{\frac{\eta u}{d}}$

Figure 12. (a) Secondary (in magnitude) term in the velocity solution for high-tide conditions, with superimposed beach profile. (b) Dominant term in the velocity solution. Contours in Figure $12 b$ describe the ratio of the primary to secondary terms.

The parameterizations of $A_{v}$ investigated appear robust to errors in the inputs describing the wave field and the mean setup. Predictive models of the mean flow require reliable models of wave transformation and setup, with well-defined model parameters. The model of Thornton and Guza [1983] is found to adequately model the wave field. Application of this model requires specification of $B$ and $\gamma$. The parameterization of $\gamma$ has been in- vestigated by Sallenger and Holman [1985] and may be estimated with only limited confidence. The parameterization of $B$ is largely unknown. The form presented here, $B=f\left(\xi_{0}\right)$, is based on very restricted data and warrants further investigation.

Similarly, the model for wave setup is poorly constrained by field data. Figure 12 shows the importance of setup as a driving term for nearshore circulation. 

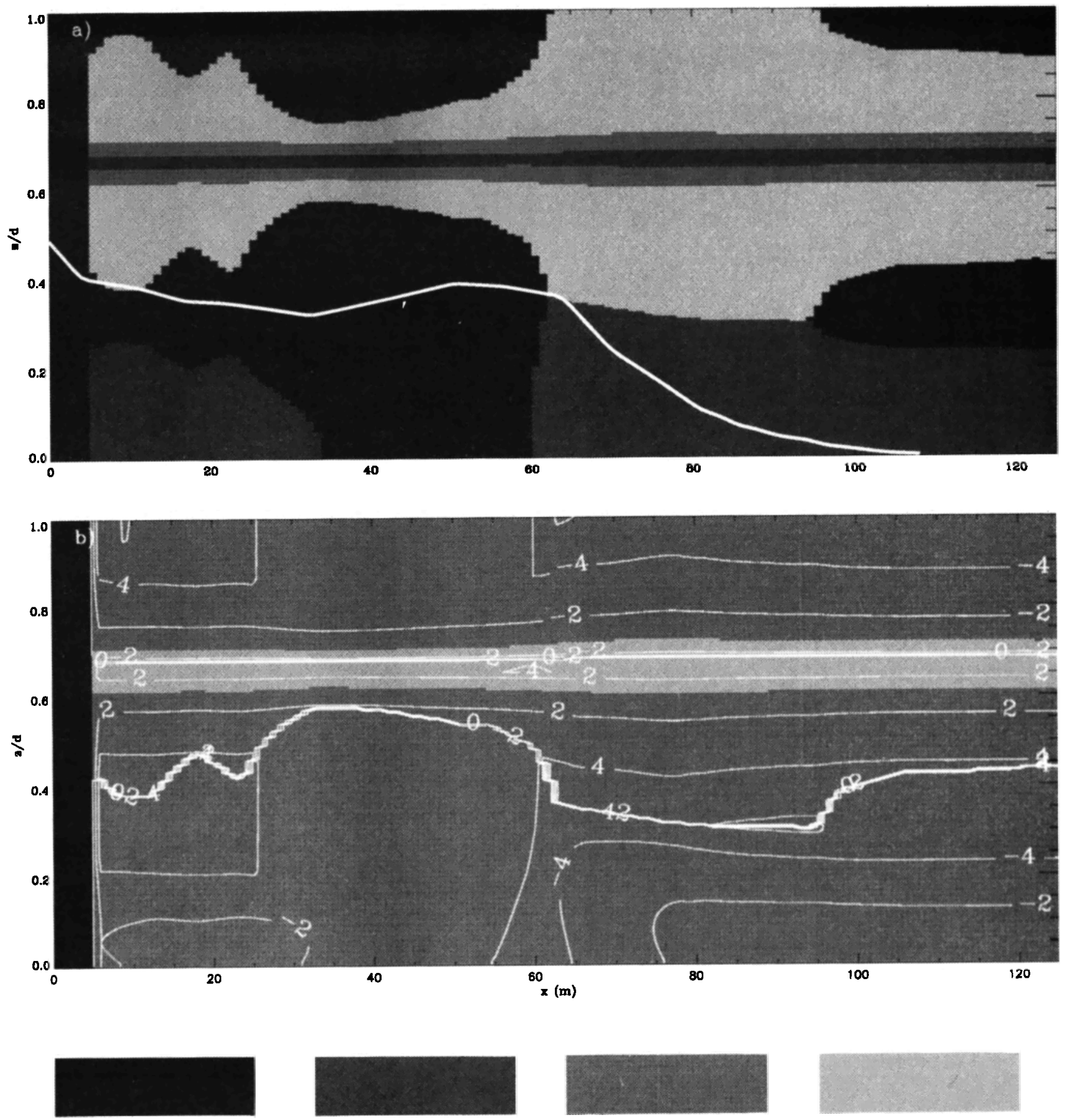

$\left(\frac{A^{*}}{2}\right)^{\prime} \frac{\sigma^{*}}{4}$

$A^{2} k \frac{6}{4}$

$g \bar{\eta}_{\mathbf{x}} \mathrm{d}^{2}$

$-2 A_{v} \frac{\bar{m}}{d}$

Figure 13. (a) Secondary (in magnitude) term in the velocity solution for low-tide conditions, with superimposed beach profile. (b) Dominant term in the velocity solution. Contours in Figure $13 \mathrm{~b}$ describe the ratio of the primary to secondary terms.

Setup is clearly the dominant forcing for the flows observed. Field verification of setup models are critical to further evaluation of modeling efforts.

The model is simplistic in that it neglects horizontal mixing and assumes $A_{v} \neq f(z, t)$. Both these assumptions demand further field investigation, and the existing data may suggest that these assumptions are wrong. Nonetheless, the current effort is largely consistent with 

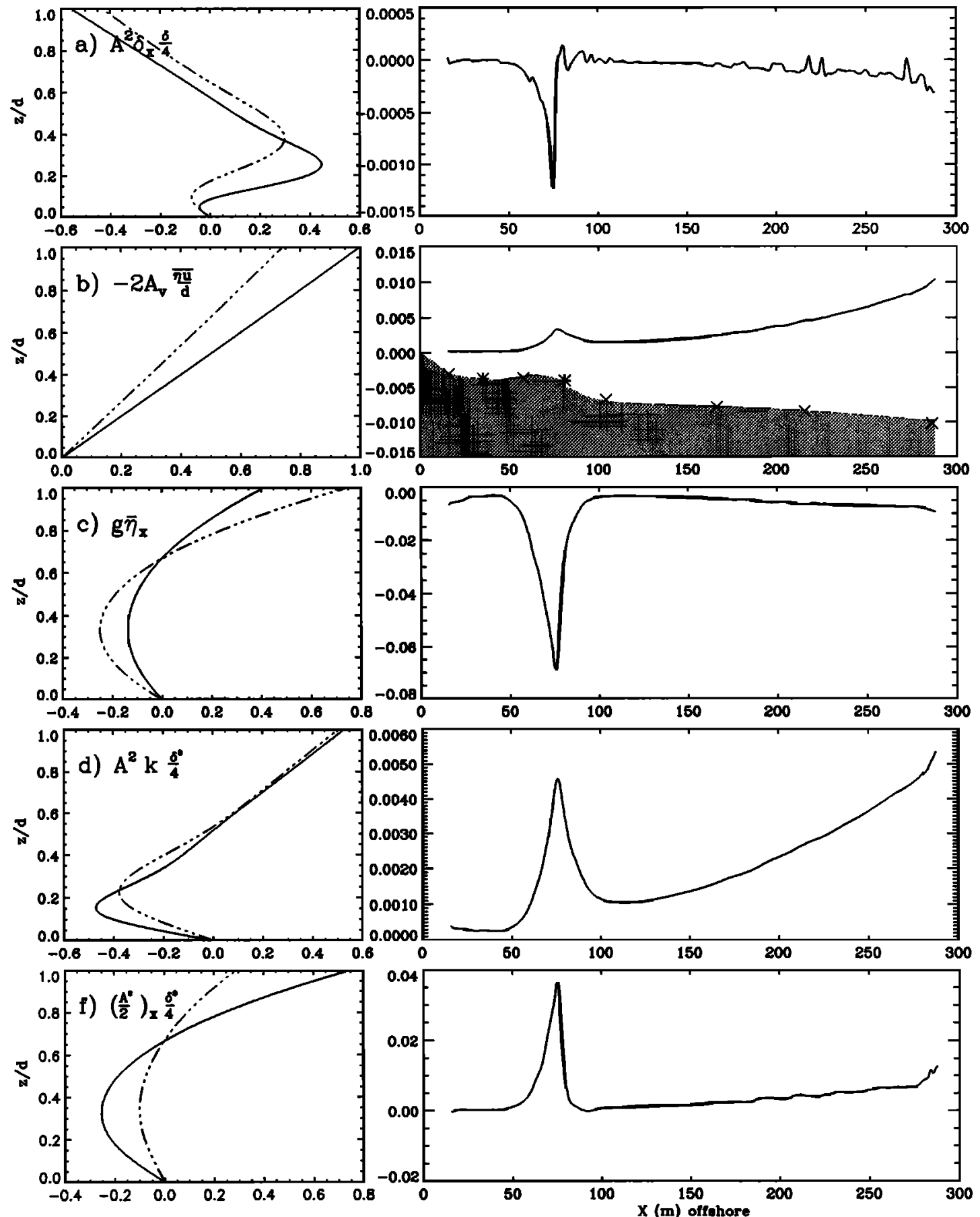

Figure 14. Spatial behavior of velocity solution, $A_{v} U$. Right-hand panels show cross-shore behavior. Figure 14b shows position of data runs for October 12. Left-hand panels show vertical behavior. Dot-dashed (solid) line shows inshore (offshore) station marked by asterisk in Figure $14 \mathrm{~b}$.

the data available. The model is largely successful in describing the interior mean flows across a complicated nearshore.

\section{Appendix 1: Wave-induced Velocities}

The turbulent boundary layer is modeled by the simplified equations of motion

$$
\begin{aligned}
u_{t} & =-\frac{1}{\rho} P_{x} \text { (interior of fluid) } \\
u_{t}-A_{v} u_{z z} & =-\frac{1}{\rho} P_{x}, \quad \text { (boundary layer) }
\end{aligned}
$$

following Russell and Osorio [1967]. The turbulent boundary layer results from the imposition of a no-slip condition at the bed in the presence of waves. This 
boundary layer, forced by waves, is not to be confused with the mean current boundary layer, which occupies the entire water column. By equating the pressure terms, $P_{x}$, in (15), we arrive at an equation for the velocity given by

$$
u_{t}=\left(u_{t}\right)_{\infty}+A_{v} u_{z z}
$$

where $\left.\right|_{\infty}$ denotes values in the fluid interior due to waves. This equation is subject to the boundary conditions

$$
\begin{aligned}
& u=0 \text { at } z=0 \\
& u=\left.u\right|_{\infty} \text { at } z=\infty,
\end{aligned}
$$

where $u_{\left.\right|_{\infty}}$ is given by equation (9). Assuming $A v \neq$ $f(z, t)$, the solution for $u$ is given by

$$
u=A\left[\cos (k x+\sigma t)-e^{-\phi} \cos (k x+\sigma t-\phi)\right],
$$

where $\phi=z^{\prime} / \delta$ and $\delta=\left(2 A_{v} / \sigma\right)^{1 / 2}$. The eddy viscosity in the boundary layer solution is the same as that in equation (4). Svendsen et al. [1987] and Deigaard et al. [1991], on the basis of laboratory observations, would suggest that the boundary layer eddy viscosity is greatly reduced relative to that seen in the fluid interior because of turbulence generated by breaking waves. Similar observations are not available for field conditions. We note that the vertical structure of the mean flow seen in the lab is different in character from the data discussed herein. In the absence of relevant field observations of the near-bed stress distribution, we choose to impose a constant eddy viscosity throughout the water column. We stress that this choice is probably incorrect. Without direct turbulence measurements, or substantially increased vertical resolution in the mean flow observations, we have insufficient data to define and verify more "realistic" models of the eddy viscosity.

The vertical velocity $w$ is given from continuity by

$$
\begin{aligned}
w_{z} & =-u_{x} \\
w & =-\int_{0}^{z^{\prime}} u_{x} \partial z^{\prime} .
\end{aligned}
$$

Thus $w$ is given by

$$
\begin{aligned}
-A_{x} \delta \quad & {[\phi \cos (k x+\sigma t)} \\
& -\frac{e^{-\phi}}{2} \cos (k x+\sigma t)(\sin \phi-\cos \phi) \\
& +\frac{e^{-\phi}}{2} \sin (k x+\sigma t)(\sin \phi+\cos \phi) \\
& \left.-\frac{1}{2}\{\cos (k x+\sigma t)+\sin (k x+\sigma t)\}\right] \\
+A k \delta \quad & {[\phi \sin (k x+\sigma t)} \\
& -\frac{e^{-\phi}}{2} \sin (k x+\sigma t)(\sin \phi-\cos \phi) \\
& -\frac{e^{-\phi}}{2} \cos (k x+\sigma t)(\sin \phi+\cos \phi) \\
& \left.-\frac{1}{2}\{\sin (k x+\sigma t)-\cos (k x+\sigma t)\}\right]
\end{aligned}
$$

$$
\begin{aligned}
&+A h_{x} \quad\left[e^{-\phi} \cos (k x+\sigma t) \cos \phi\right. \\
&\left.+e^{-\phi} \sin (k x+\sigma t) \sin \phi-\cos (k x+\sigma t)\right] \\
&-A \delta_{x} \quad[ \phi e^{-\phi} \cos (k x+\sigma t) \cos \phi \\
&+\phi e^{-\phi} \sin (k x+\sigma t) \sin \phi \\
&-\frac{e^{-\phi}}{2} \cos (k x+\sigma t)(\sin \phi-\cos \phi) \\
&+\frac{e^{-\phi}}{2} \sin (k x+\sigma t)(\sin \phi+\cos \phi) \\
&\left.-\frac{1}{2}\{\cos (k x+\sigma t)+\sin (k x+\sigma t)\}\right] .
\end{aligned}
$$

\section{Appendix 2: Wave Transformation Model}

The wave height transformation model is based on an energy flux balance. Energy losses associated with wave breaking are modeled using observed breaking wave heights coupled with a periodic bore dissipation model. The model is given by

$$
\frac{\partial E C_{g x}}{\partial x}=\left\langle\epsilon_{b}\right\rangle
$$

where $E$ is the energy density, $C_{g x}$ is the $x$ component of the group velocity and $\left\langle\epsilon_{b}\right\rangle$ is the bore dissipation. The energy density is given, to lowest order, by

$$
E=\frac{1}{8} \rho g H_{r m s}^{2},
$$

and the group velocity is

$$
C_{g x}=\frac{C}{2}\left(1+\frac{2 k h}{\sinh 2 k h}\right) \cos \bar{\theta},
$$

where $\bar{\theta}$ is the mean wave direction (here assumed to be 0 ) associated with the average frequency corresponding to the peak of the spectrum. The root mean square wave height, $H_{r m s}$, is calculated from observed velocities as

$$
H_{r m s}=2 \sqrt{2}\left\{\int_{0.05}^{0.33}|H(f)|^{2}\left[G_{u}(f)+G_{v}(f)\right] d f\right\}^{\frac{1}{2}},
$$

where $G_{u}(f)$ is the cross-shore flow spectrum, $G_{v}(f)$ is the alongshore flow spectrum, and $H(f)$ is the linear transformation function given by

$$
H(f)=\frac{\sinh |k| h}{\sigma \cosh (|k|(h+z))} .
$$

Describing the probability function of breaking wave heights, $p_{b}(H)$, as a weighting of the Rayleigh distribution for all waves, $p(H)$, such that

$$
p_{b}(H)=W(H) p(H),
$$

where 


$$
W(h)=\left(\frac{H_{\mathrm{rms}}}{\gamma h}\right)^{n}\left[1-\exp \left(-\left(\frac{H}{\gamma h}\right)^{2}\right)\right] \leq 1
$$

and $n=2$, results in a form for the dissipation of

$$
\left\langle\epsilon_{b}\right\rangle=\frac{3 \sqrt{\pi}}{16} \rho g B^{3} \bar{f} \frac{H_{r m s}^{5}}{\gamma^{2} h^{3}}\left[1-\frac{1}{\left(1+\left(\frac{H_{r m s}}{\gamma h}\right)^{2}\right)^{\frac{5}{2}}}\right] .
$$

In equation (28), $\bar{f}$ is the average frequency corresponding to the peak of the spectrum and $\gamma$ is an adjustable coefficient given by

$$
H_{r m s}=\gamma h .
$$

Values for $\gamma$ are determined from the sled data, following Sallenger and Holman [1985]. Best fit values are shown in Figure 15 for all runs. The data clusters into two groups. Data on the outer flank of the bar are described by a larger value of $\gamma$. This is suggestive of a zone where breaking waves, propagating over rapidly varying topography, cannot achieve the saturated conditions prescribed by a simple model, $H=\gamma h$. A value of $\gamma=0.34$ will be used in subsequent application of the wave transformation model.

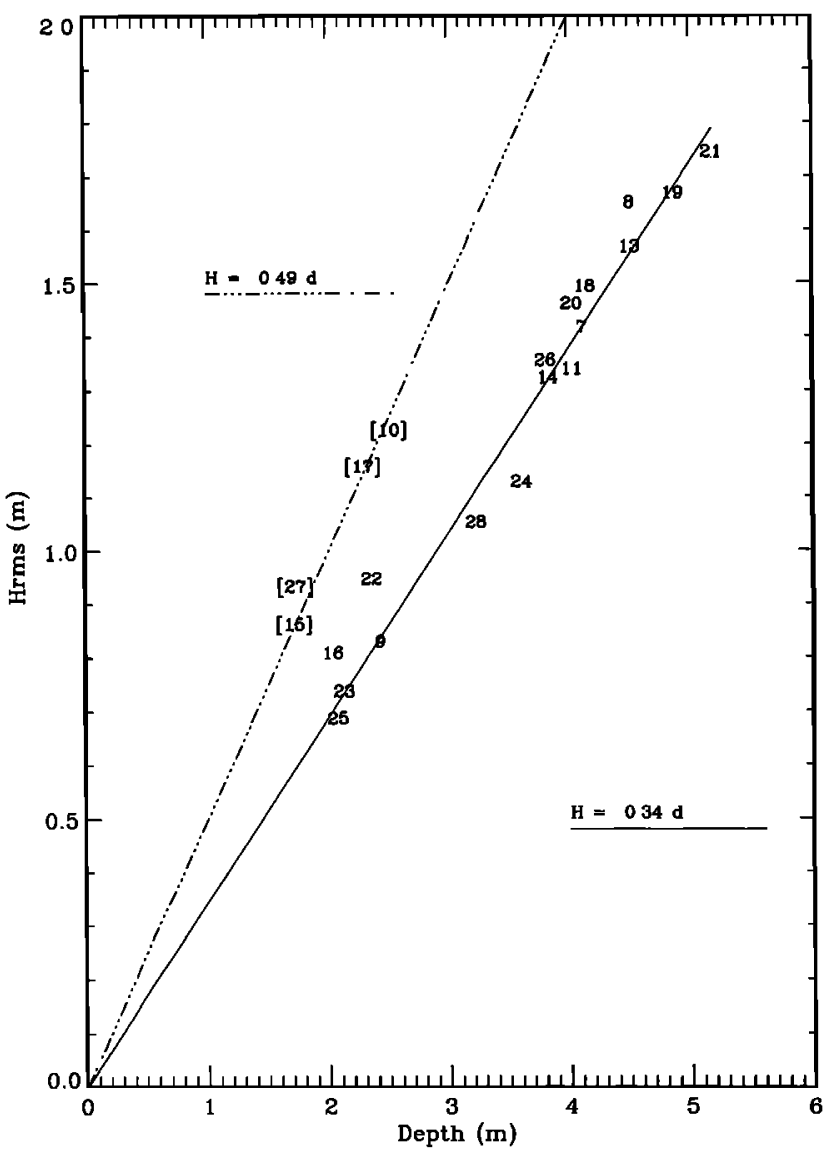

Figure 15. Regression of $H$ against $h$ for all data. Separate regressions are shown for runs on the outer bar flank (denoted by []) and all other data runs.
Sallenger and Holman [1985] also suggested (using the same data set examined here) that $\gamma$ was a function of the local beach slope $\beta$. A. Bowen (personal communication, 1992) has found that incorporating $\gamma=f(\beta)$ in the model of Thornton and Guza [1983] reduces the variability in $B$ required to reproduce observed wave heights. This approach, applied to this data set, did not result in more stable estimates of $B$.

Acknowledgments. The modeling effort was initiated by Tony Bowen many years past, and we are grateful for his generous sharing of ideas and experience. We acknowledge that he would have done a better job. We also thank the anonymous reviewers and the AGU editorial staft for their thoughtful and careful contributions. This work was funded by the U.S. Geological Survey, with funding and logistics support for the field effort from the U.S. Army Engineer Waterways Experiment Station, Coastal Engineering Research Center, Vicksburg, Miss.

\section{References}

Battjes, J., Set-up due to irregular waves, Proc. 13th Int. Conf. Coastal Eng., 1933-2004, 1972.

Boreki, O., Distribution of wave--induced momentum fluxes over depth and application within the surf sone, Ph.D. thesis, Univ. of Del., Newark, 1982.

Bowen, A., Simple models of nearshore sedimentation; beach profiles and longshore bars, in The Coastline of Canada, edited by S. McCann, Pap. Geol. Surv. Can., 80-10, 1-11, 1980.

Bowen, A., D. Inman, and V. Simmons, Wave 'set-down' and setup, J. Geophys. Res., 73, 2569-2577, 1968.

Collins, J., Probabilities of breaking wave characteristics, Proc. 14th Int. Conf. Coastal Eng., 399-412, 1974.

Dally, W., A numerical model for beach profile evolution, Master's thesis, Univ. of Del., Newark, 1980.

Dally, W., and R. Dean, Suspended sediment transport and beach profile evolution, J. Water. Port Coastal and Ocean Div. Am. Soc. Civ. Eng., 110(1), 15-33, 1984.

Deigaard, R., P. Justesen, and J. Fredsoe, Modelling of undertow by a one-equation turbulence model, Coastal Eng., 15, 431-458, 1991.

DeVriend, H. J., and M. J. F. Stive, Quasi-3d modelling of nearshore currents, Coastal Eng., 11, 565-602, 1987.

Guza, R., and E. Thornton, Local and shoaled comparisons of sea surface elevations, pressures, and velocities, J. Geophys. Res., 85, 1524-1530, 1980.

Hansen, J., and I. Svendsen, A theoretical and experimental study of undertow, Proc. 19th Int. Conf. Coastal Eng., 2246-2262, 1984.

Longuet-Higgins, M., Mass transport in water waves, Philos. Trans. R. Soc. London, 245(903), 535-581, 1953.

Longuet-Higgins, M., and R. Stewart, Radiation stress in water waves: A physical discussion with applications, Deep Sea Res., 11, 529-562, 1964.

Okayasu, A., T. Shibayama, and K. Horikawa, Vertical variations of undertow in the surf zone, Proc. 21st Int. Conf. Coastal Eng., 478-491, 1988.

Russell, R., and J. Osorio, An experimental investigation of drift profiles in a closed channel, Proc. 6th Int. Conf. Coastal Eng., 171-183, 1967.

Sallenger, A. H., Jr., and R. A. Holman, Wave energy saturation on a natural beach of variable slope, J. Geophys. Res., 90, 11,939-11,944, 1985. 
Sallenger, A. H., Jr., R. Holman, and W. Birkemeier, Storminduced response of a nearshore-bar system, Mar. Geol., 64, 237-257, 1985.

Sallenger, A., P. Howard, C. Fletcher, and P. Howd, A system for measuring bottom profile, waves and currents in the high-energy nearshore environment, Mar. Geol., 64, 237-257, 1983.

Shepard, F., and E. LaFond, Undertow, Science, 89, 1-2, 1939.

Stive, M., and H. Wind, Cross-shore mean flow in the surf zone, Coastal Eng., 10, 325-340, 1986.

Svendsen, I., A. Hemming, and J. Buhr Hansen, The interaction between the undertow and the boundary layer flow on a beach, J. Geophys. Res., 92, 11,845-11,856, 1987.

Svendsen, I., and R. Lorenz, Velocities in combined undertow and longshore currents, Coastal Eng., 13, 55-79, 1989.
Thornton, E. B., and R. T. Guza, Transformation of wave height distribution, J. Geophys. Res., 88, 5,925-5,938, 1983.

Thornton, E., Variation of longhshore currents across the surfzone, Proc. 12th Int. Conf. Coastal Eng., 291-308, 1970.

J. W. Haines and A. H. Sallenger, Jr. U.S. Geological Survey, Center for Coastal Geology, 600 Fourth Street South, St. Petersburg, FL 33701. (e-mail: Internet:jhaines@wayback.er.usgs.gov, asallenger@wayback.er.usgs.gov)

(Received October 28, 1992; revised February 14, 1994; accepted February 14, 1994.) 